Washington University School of Medicine Digital Commons@Becker

Open Access Publications

2009

\title{
An electrostatic switch displaces phosphatidylinositol phosphate kinases from the membrane during phagocytosis
}

\author{
Gregory D. Fairn \\ Hospital for Sick Children - Toronto \\ Koji Ogata \\ Hospital for Sick Children - Toronto \\ Roberto J. Botelho \\ Ryerson University \\ Philip D. Stahl \\ Washington University School of Medicine in St. Louis \\ Richard A. Anderson \\ University of Wisconsin Medical School
}

See next page for additional authors

Follow this and additional works at: https://digitalcommons.wustl.edu/open_access_pubs

Part of the Medicine and Health Sciences Commons

Please let us know how this document benefits you.

\section{Recommended Citation}

Fairn, Gregory D.; Ogata, Koji; Botelho, Roberto J.; Stahl, Philip D.; Anderson, Richard A.; De Camilli, Pietro; Meyer, Tobias; Wodak, Shoshana; and Grinstein, Sergio, "An electrostatic switch displaces phosphatidylinositol phosphate kinases from the membrane during phagocytosis." The Journal of Cell Biology. 187, 5. 701-714. (2009).

https://digitalcommons.wustl.edu/open_access_pubs/559

This Open Access Publication is brought to you for free and open access by Digital Commons@Becker. It has been accepted for inclusion in Open Access Publications by an authorized administrator of Digital Commons@Becker. For more information, please contact vanam@wustl.edu. 


\section{Authors}

Gregory D. Fairn, Koji Ogata, Roberto J. Botelho, Philip D. Stahl, Richard A. Anderson, Pietro De Camilli, Tobias Meyer, Shoshana Wodak, and Sergio Grinstein 


\title{
An electrostatic switch displaces phosphatidylinositol phosphate kinases from the membrane during phagocytosis
}

\author{
Gregory D. Fairn, ${ }^{1}$ Koji Ogata, ${ }^{2}$ Roberto J. Botelho, ${ }^{3}$ Philip D. Stahl, ${ }^{4}$ Richard A. Anderson, ${ }^{5}$ Pietro De Camilli, $6,7,8,9$ \\ Tobias Meyer, ${ }^{10}$ Shoshana Wodak, ${ }^{2}$ and Sergio Grinstein ${ }^{1}$ \\ IProgram in Cell Biology and ${ }^{2}$ Structural Biology Program, Hospital for Sick Children, Toronto, Ontario, Canada M5G1X8 \\ ${ }^{3}$ Department of Chemistry and Biology, Ryerson University, Toronto, Ontario, Canada M5B 2K3 \\ ${ }^{4}$ Department of Cell Biology and Physiology, Washington University School of Medicine, St. Louis, MO 63110 \\ ${ }^{5}$ Program in Molecular and Cellular Pharmacology, Department of Pharmacology, University of Wisconsin Medical School, Madison, WI 53706 \\ ${ }^{6}$ Department of Cell Biology, ${ }^{7}$ Department of Neurobiology, ${ }^{8}$ Howard Hughes Medical Institute, and ${ }^{9}$ Program in Cellular Neuroscience, Neurodegeneration, and Repair, \\ Kavli Institute of Neuroscience, Yale University School of Medicine, New Haven, CT 06510 \\ ${ }^{10}$ Department of Chemical and Systems Biology, Stanford University School of Medicine, Stanford, CA 94305
}

$\mathrm{P}$ lasmalemmal phosphatidylinositol (PI) 4,5-bisphosphate $\left(\mathrm{PI} 4,5 \mathrm{P}_{2}\right)$ synthesized by $\mathrm{PI}$ 4-phosphate (PI4P) 5-kinase (PIP5K) is key to the polymerization of actin that drives chemotaxis and phagocytosis. We investigated the means whereby PIP5K is targeted to the membrane and its fate during phagosome formation. Homology modeling revealed that all PIP5K isoforms feature a positively charged face. Together with the substratebinding loop, this polycationic surface is proposed to constitute a coincidence detector that targets PIP5Ks to the plasmalemma. Accordingly, manipulation of the surface

\section{Introduction}

Phagocytosis, a central event in the elimination of foreign and apoptotic bodies, is initiated by the interaction of ligands on target particles with receptors on the surface of phagocytic cells. Through a series of intricate events that are incompletely understood, the activated receptors trigger the localized assembly of actin, which propels the extension of pseudopods that surround and ultimately engulf the particle. Completion of the process and detachment of the phagosomal vacuole from the surface membrane require the subsequent depolymerization of actin (O'Reilly et al., 2003; Scott et al., 2005).

Correspondence to Sergio Grinstein: sergio.grinstein@sickkids.ca

Abbreviations used in this paper: FKBP, FK506-binding protein; FRB; FK506 rapamycin-binding domain; PC, phosphatidylcholine; PH, pleckstrin homology; $\mathrm{PI}$, phosphatidylinositol; PI4,5P2, PI 4,5-bisphosphate; PI4P, PI 4-phosphate; PIP4K, PI 5-phosphate 4-kinase; PIP5K, PI4P 5-kinase; PM-RFP, palmitoylated/ myristoylated-tagged RFP; PS, phosphatidylserine; R-Pre, arginine and prenylatedtagged RFP. charge displaced PIP5Ks from the plasma membrane. During particle engulfment, PIP5Ks detached from forming phagosomes as the surface charge at these sites decreased. Precluding the change in surface charge caused the PIP5Ks to remain associated with the phagosomal cup. Chemically induced retention of PIP5K- $\gamma$ prevented the disappearance of $\mathrm{PI} 4,5 \mathrm{P}_{2}$ and aborted phagosome formation. We conclude that a bistable electrostatic switch mechanism regulates the association/dissociation of PIP5Ks from the membrane during phagocytosis and likely other processes.

The dynamic rearrangement of actin during phagocytosis is tightly linked to the metabolism of phosphatidylinositol (PI) 4,5-bisphosphate (PI4,5P $\mathrm{P}_{2}$ ). As in other cells (Stauffer et al., 1998; Várnai and Balla, 1998), this phosphoinositide is abundant in the inner leaflet of the plasma membrane of unstimulated phagocytes (Botelho et al., 2000). However, at the onset of phagocytosis, the phosphoinositide accumulates further at sites where pseudopods are formed. This localized increase in $\mathrm{PI} 4,5 \mathrm{P}_{2}$ likely serves as a platform for the robust actin polymerization required for pseudopod extension. Two types of interactions underlie the effects of $\mathrm{PI} 4,5 \mathrm{P}_{2}$ : stereospecific binding of proteins with domains that selectively recognize its headgroup and electrostatic interactions of polycationic

(c) 2009 Fairn et al. This article is distributed under the terms of an AttributionNoncommercial-Share Alike-No Mirror Sites license for the first six months after the publication date (see http://www.jcb.org/misc/terms.shtml). After six months it is available under a Creative Commons License (Attribution-Noncommercial-Share Alike 3.0 Unported license, as described at http://creativecommons.org/licenses/by-nc-sa/3.0/). 
proteins with the negative surface charge that $\mathrm{PI} 4,5 \mathrm{P}_{2}$ confers to the membrane.

During later stages of particle engulfment, $\mathrm{PI} 4,5 \mathrm{P}_{2}$ disappears from the base of the phagocytic cup, a process that has been attributed to hydrolysis by PLC and to phosphorylation by PI 3-kinases (Araki et al., 1996). The elimination of the phosphoinositide is accompanied by actin depolymerization and is felt to be essential for phagosome closure and scission (Scott et al., 2005). Indeed, excessive and/or untimely production of $\mathrm{PI} 4,5 \mathrm{P}_{2}$ was reported to preclude actin depolymerization and inhibit particle engulfment (Scott et al., 2005). Clearly, coordination of the processes that generate and eliminate PI4,5 $\mathrm{P}_{2}$ is key to successful completion of phagocytosis. Yet it is not clear whether PI4,5 $\mathrm{P}_{2}$ synthesis is arrested during the secondary phase of phagocytosis or proceeds concomitantly but is outstripped by the degradative processes. The main objective of this study was to assess the fate of the PI4,5 $\mathrm{P}_{2}$-generating enzymes during the course of phagosome formation.

Metazoans produce PI4,5 $\mathrm{P}_{2}$ by two distinct pathways. The type I PI 4-phosphate (PI4P) 5-kinase (PIP5K) is responsible for the primordial or classical pathway, which is used by all eukaryotes. The $\alpha, \beta$, and $\gamma$ isoforms of this enzyme catalyze the phosphorylation of PI4P to PI4,5 $\mathrm{P}_{2}$. The second or alternative pathway synthesizes PI4,5P $\mathrm{P}_{2}$ from PI 5-phosphate using the type II kinase (PI 5-phosphate 4-kinase [PIP4K]; Rameh et al., 1997). These enzymes are related, as they display $30 \%$ sequence identity; however, they use different substrates and localize to different subcellular compartments. Through the generation of chimeric constructs, previous work established that an $\sim 25$-amino acid segment called the activation loop influences the differential substrate specificity and subcellular localization of the type I and II enzymes (Kunz et al., 2000). A single mutation (E362A) in the activation loop of the type I- $\beta$ kinase altered its substrate selectivity and caused it to be displaced from the plasma membrane (Kunz et al., 2002). These results suggest that recognition of PI4P is necessary, although not necessarily sufficient, for the proper plasmalemmal localization of the type I PIP5K.

It is becoming increasingly apparent that most proteins are guided to their biological destination not by one, but by multiple targeting determinants. The coexistence of multiple determinants provides the basis of a concerted detection process that ensures the specificity of protein targeting within the cell. In this study, we demonstrate that, in addition to the activation loop, PIP5K isoforms rely on at least one other determinant for their targeting to the plasma membrane. Specifically, we found that PIP5K- $\alpha,-\beta$, and $-\gamma$ feature a positively charged region on the protein surface that is essential for their association with the inner leaflet of the plasmalemma. Moreover, we report that acute and localized changes in the surface charge of the plasma membrane release the kinases from the base of forming phagosomes, terminating the synthesis of $\mathrm{PI} 4,5 \mathrm{P}_{2}$ and accentuating its disappearance at the site where its degradation is most active. Finally, we demonstrate that when the electrostatic switch that releases PIP5K from phagosomes is disabled, the kinase remains associated with nascent phagosomes that therefore fail to seal, aborting the engulfment process.

\section{Results}

Expression and localization of PIP5K

isoforms in RAW macrophages

The purpose of our experiments was to investigate the fate of PIP5K during phagosome formation and maturation. This required identifying the isoforms of PIP5K that are expressed in macrophages. Immunoblot analysis using isoform-specific antibodies revealed that RAW murine macrophages express the PIP5K- $\alpha,-\beta$, and $-\gamma$ isoforms (Fig. 1 a). It is not clear whether the presence of multiple bands in the immunoblots of PIP5K- $\beta$ and $-\gamma$ reflects the existence of splice variants or the occurrence of posttranslational modifications. Next, we used spinning-disc confocal microscopy to visualize the localization of the different isoforms. Because available antibodies are not adequate for immunolocalization of all of the endogenous PIP5K isoforms, the RAW cells were transfected with GFP- or YFP-tagged versions of the individual isoforms. We deliberately chose to analyze only cells displaying low to medium levels of fluorescence, to minimize complications caused by overexpression. As illustrated in Fig. $1 \mathrm{c}$, the $\alpha$ and $\beta$ isoforms, as well as the 90- and $87-\mathrm{kD}$ splice variants of the $\gamma$ isoform, were found predominantly at the plasma membrane, which was identified by coexpression of a palmitoylated/myristoylated-tagged RFP (PM-RFP) that has been used extensively as a plasmalemmal marker (Teruel et al., 1999). As expected, the distribution of the PIP5K isoforms was found to coincide with that of their product, $\mathrm{PI} 4,5 \mathrm{P}_{2}$, which was detected using the pleckstrin homology (PH) domain of PLC- $\delta$ (Fig. 1 b). In some instances, a fraction of the fluorescence was found in intracellular punctate structures (Fig. 1, b and c), which are identified as endosomes (not depicted). The fraction of PIP5K reported to exist in the nucleus of other cell types (Payrastre et al., 1992; Boronenkov et al., 1998) was not detectable in RAW macrophages by the methods used.

Homology modeling the structure of PIP5K The activation loop of the PIP5Ks is important in targeting the isoforms to the plasma membrane (Kunz et al., 2000). However, it is becoming increasingly clear that most proteins are recruited to their specific sites of action in the cell by multiple targeting determinants. Indeed, the crystal structure of the related enzyme, PIP4K, revealed the presence of a positively charged region on the protein surface that is thought to contribute to its recruitment to membranes by electrostatic means (Burden et al., 1999). Therefore, we analyzed the structure of the $\alpha, \beta$, and $\gamma$-90 isoforms of PIP5K in search for additional targeting determinants. Although the X-ray structure of the PIP5Ks has not been determined, the functional and sequence similarity between these enzymes and PIP4K warranted approximation of their structure by homology-modeling techniques using the PIP4K structure as a template (see Materials and methods). Atomic models for the three PIP5K variants were thus built on the basis of the known 3-Å resolution crystal structure of PIP4K (Protein Data Bank [PDB] accession no. 1bo1; Rao et al., 1998).

The validity of the models was checked by using Ramachandran plots and Procheck software (as described in Materials 


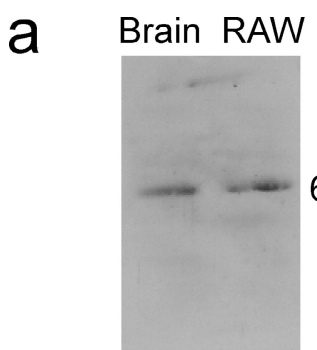

PIP5K $\alpha$

b
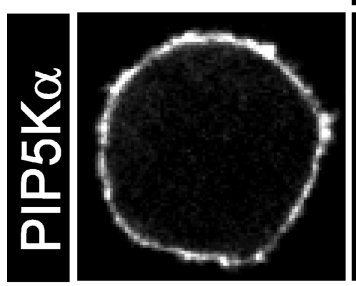

$\frac{a}{2}$

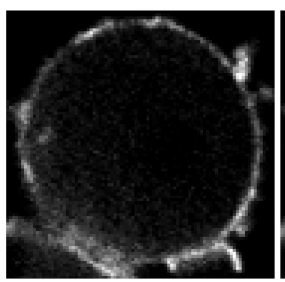

$n$
$\frac{1}{2}$
$\frac{1}{n}$
$\frac{0}{0}$
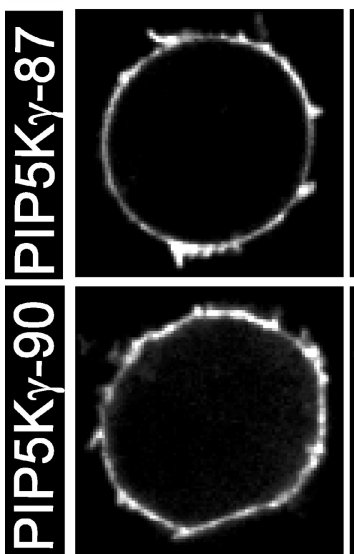

$68 \mathrm{kD}$
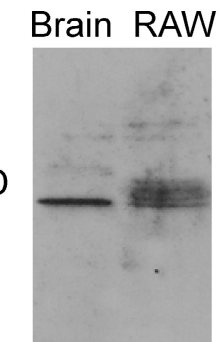

PIP5K $\beta$

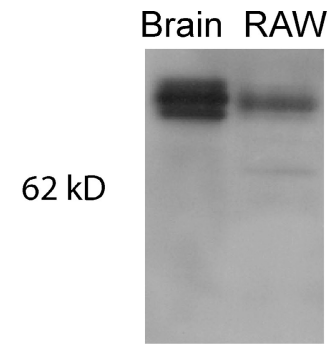

PIP5K $\gamma$
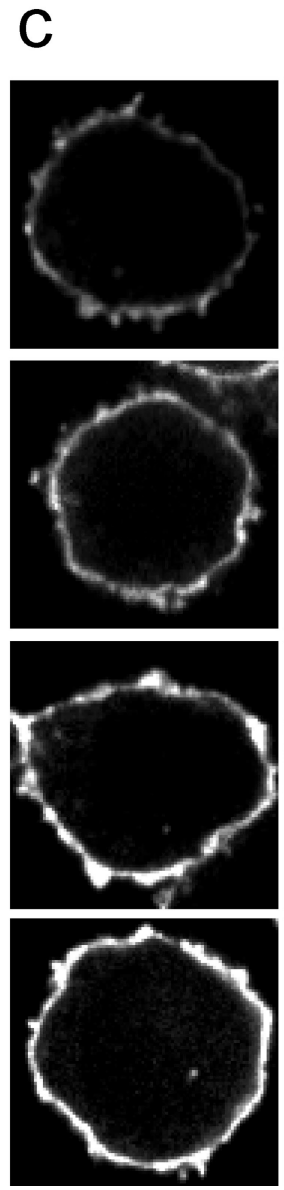

$87 \mathrm{kD}$

\section{PM-RFP}
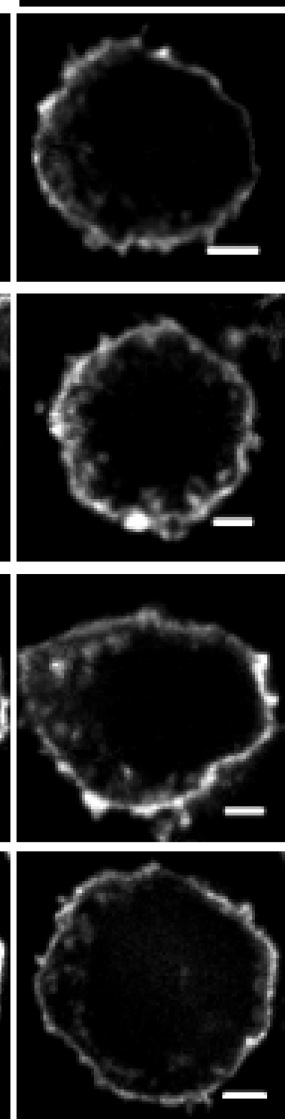

Figure 1. Expression and localization of PIP5Ks in RAW cells. (a) Immunoblots of RAW cell and brain extracts probed with PIP5K isoform-specific antibodies. (b and c) Colocalization of PIP5K isoforms with their product, $\mathrm{PI} 4,5 \mathrm{P}_{2}$, at the plasma membrane. RAW cells were transiently cotransfected with GFP or YFP chimeras of the PIP5K isoforms and with either RFP-PH-PLC- $\delta$ (b) or PM-RFP used as a plasmalemmal marker (c). The distribution of the fluorescent proteins was analyzed by spinningdisc confocal microscopy, and representative images acquired near the middle of the cell are illustrated. Bars, 3 rm. and methods; Laskowski et al., 1993). The Ramachandran plots (Fig. S1) for the PIP4K structure and PIP5K models show similar distribution of dihedral angles of the amino acids with few outliers. Alignment of the protein sequences of PIP4K and the three PIP5Ks demonstrates a conservation of the catalytic triad composed of a lysine and two aspartic acid residues (Fig. S2). The three-dimensional model of the catalytic region of PIP5K- $\alpha$ shows the same geometry for the catalytic triad as in the PIP4K structure (Fig. S3). Together, these results suggest that the models generated are of good quality.

Fig. 2 compares the surface electrostatic properties of the three homology-built models of PIP5K with those of the crystal structure of PIP4K. In Fig. 2 a, the PIP4K homodimer is shown with the flat face, which is thought to interact with the membrane, facing the observer. This face displays a prominent patch of positive electrostatic potential near the PIP4K dimer interface (Rao et al., 1998). The positive potential is believed to play a role in orienting this face of the protein toward the negatively charged phospholipid membrane (Rao et al., 1998; Burden et al., 1999). In Fig. 2 (b-d), the PIP5K isoforms are shown in an equivalent orientation. All three modeled PIP5K structures display a predominantly positive surface electrostatic potential on the equivalent face of the protein. Unlike PIP4K, the patch of positive surface charge is not concentrated near the dimer interface but appears more widely spread across the face of the PIP5K proteins. The predominantly positive potential of this face is better appreciated when the structures of the four proteins are rotated $90^{\circ}$ (Fig. 2, e-h). This orientation contrasts the preferential distribution of negative surface electrostatic potential on the dorsal (upward pointing) face of the proteins with that of the positive potential on the ventral face. This visual impression is confirmed by computation of the dipole moments of the proteins, represented graphically in Fig. $2(\mathrm{e}-\mathrm{h})$ by yellow arrows. The magnitude of the dipole moment calculated for PIP5K- $\alpha,-\beta$, and $-\gamma 90$ $(2,478,2,238$, and 2,944 Debye, respectively) is very similar 

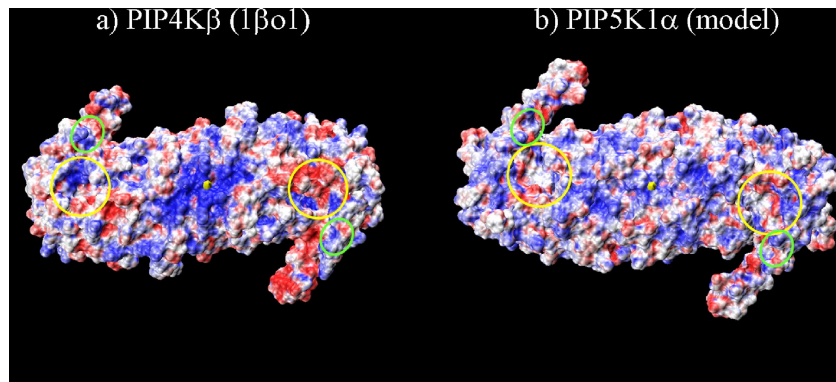

c) PIP5K1 $\beta$ (model)
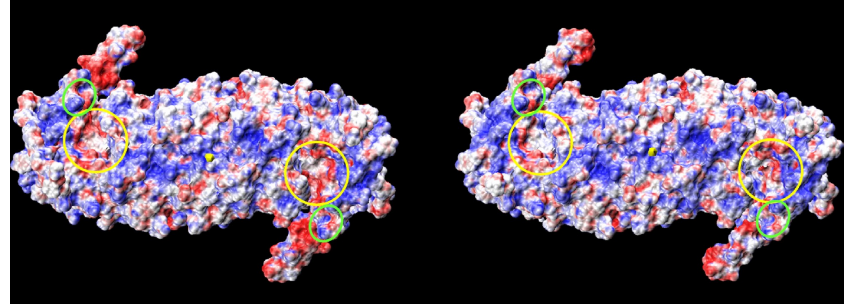

e) $\operatorname{PIP} 4 K \beta(1 \beta \circ 1)$

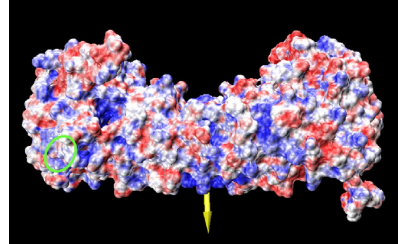

f) PIP5K1 $\alpha$ (model)

g) PIP5K1 $1 \beta$ (model)

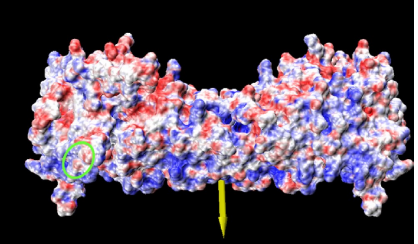

h) PIP5K1 $\gamma($ model)

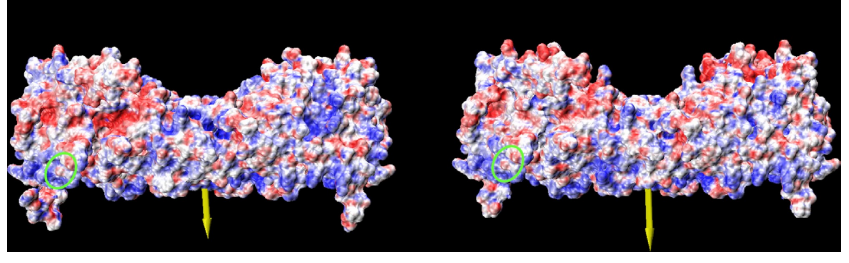

Figure 2. Structure of type II- $\beta$ PIP4K and models of type I PIP5K- $\alpha,-\beta$, and $-\gamma$ isoforms. (a-d) The reported structure of type II- $\beta$ PIP4K (a) and the predicted structures of the PIP5K- $\alpha,-\beta$, and $-\gamma 90$ isoforms deduced by homology modeling are shown in an equivalent orientation with their putative membrane-interacting face pointing toward the observer. The surfaces are colored according to the range of electrostatic potential (red, $<-10.0 \mathrm{kT} / \mathrm{e}$; blue, $>10.0 \mathrm{kT} / \mathrm{e}$ [where $\mathrm{k}=$ Boltzmann constant, $\mathrm{T}=$ absolute temperature, and $e=$ electron]). The electrostatic surface potentials were computed using the continuum solvation model embodied in the Poisson-Boltzmann method, and the adaptive Poisson-Boltzmann solver was implemented in the APBS software. (e-h) The proteins are shown in an orientation corresponding to a $90^{\circ}$ rotation from the orientations in a-d such that the presumed membrane-associated face of the proteins points downward. The ATP-binding site and a reported phosphorylation site are highlighted by yellow and green circles, respectively. The dipole moments (yellow arrows) were calculated directly from the atomic models and the atom partial charges using Protein Dipole Moments Server. The magnitude of the arrows is directly proportional to the strength on the dipole (Debye).

to that calculated for the PIP4K template (2,643 Debye). The tendency of the ventral face of PIP5K- $\alpha,-\beta$, and $-\gamma 90$ to interact with negatively charged membranes suggests that electrostatic attraction contributes to the targeting of these isoforms to the plasma membrane, which is the most negatively charged surface exposed to cytosolic proteins.
Purified PIP5Ks bind to

anionic phospholipids

The structures predicted by molecular modeling suggest that PIP5K isoforms might bind preferentially to anionic membranes. We initially tested this prediction in vitro, measuring the ability of two recombinant kinases to bind to immobilized lipids. Bacterially expressed and purified GST fusions of full-length PIP5K- $\alpha$ and $-\gamma 87$ were overlaid on dot blots of multiple lipids of varying charge. As shown in Fig. 3 a, both isoforms tested bound exclusively to anionic lipids, including phosphoinositides, phosphatidylserine (PS), phosphatidic acid, and cardiolipin but not to zwitterionic or neutral lipids such as phosphatidylethanolamine or cholesterol, respectively. When expressed by itself, GST failed to associate with any of the lipids on the dot blot (Fig. 3 a), implying that binding of the GST kinase fusions to anionic lipids was specific. It is noteworthy that, among the phosphoinositides, both kinases bound preferentially to their substrate, PI4P. However, the preference was not absolute, and binding of both kinases to $\mathrm{PI} 4,5 \mathrm{P}_{2}$ and $\mathrm{PI} 3,4,5 \mathrm{P}_{3}$ was readily apparent. Because it was of the same magnitude as that seen for other anionic lipids, such binding is likely to be electrostatic. These findings are consistent with previous results that demonstrated that murine PIP5K- $\beta$ binds to PI4,5 $\mathrm{P}_{2}$ and PI3,4,5 $\mathrm{P}_{3}$ in addition to phosphatidic acid (Jarquin-Pardo et al., 2007).

The binding properties of PIP5K- $\alpha$ and the relative contribution of the substrate and of anionic lipids were also analyzed using hydrophobic (C18 derivatized) beads coated with individual phospholipids or with combinations thereof (Fig. 3 b). As found for the dot blots, PIP5K- $\alpha$ bound preferentially to anionic lipids; a mixture of $80 \%$ phosphatidylcholine (PC) with $20 \%$ PS (approximating the mole ratio thought to exist in the inner leaflet of the membrane) was severalfold more effective than $\mathrm{PC}$ alone. The effect was even more pronounced when $2 \% \mathrm{PI} 4,5 \mathrm{P}_{2}$ and phosphatidic acid were also added to mimic the composition of the inner monolayer of the plasma membrane. PI4P also promoted PIP5K- $\alpha$ binding in a concentration-dependent manner. Of note, a synergistic effect was noted when physiological concentrations of PI4P and anionic lipids were presented together (Fig. 3 B).

\section{Role of phosphoinositides in PIP5K localization}

Previous work suggested that substrate recognition is important for proper subcellular targeting of the type I and II PIPK kinases. The substrate for type I kinases, PI4P, is found predominantly in the plasma membrane and Golgi complex (Roy and Levine, 2004; D'Angelo et al., 2008; Hammond et al., 2009). In the Golgi, PI4P serves as a targeting determinant for proteins involved in lipid metabolism, including the ceramide transfer protein, CERT, the oxysterol-binding protein, OSBP, and the glucosylceramide transfer protein, FAPP2, via their PH domains (Godi et al., 2004; Tóth et al., 2006). However, despite the presence of PI4P in the Golgi, the PIP5K isoforms are not detectable in this organelle in RAW macrophages and in other cells and instead localize predominantly to the plasma membrane. This suggests that substrate recognition is not the sole determinant of the subcellular localization of PIP5K. The strong dipole moment revealed by homology modeling, together with the in vitro binding data, suggests that the 
a

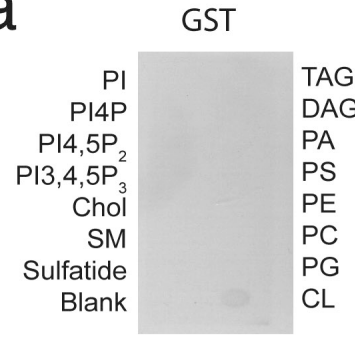

b
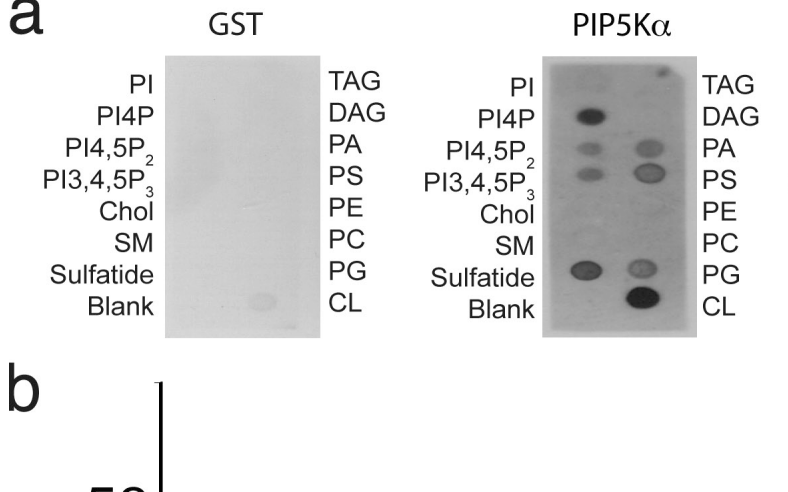

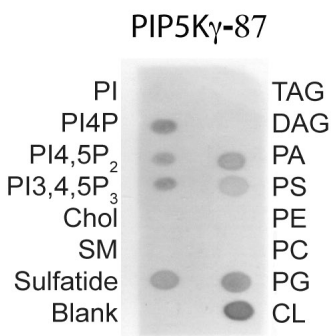

$20 \%$ PS

$2 \%$ PIP2

$2 \%$ PA

$0.5 \%$ PI4P

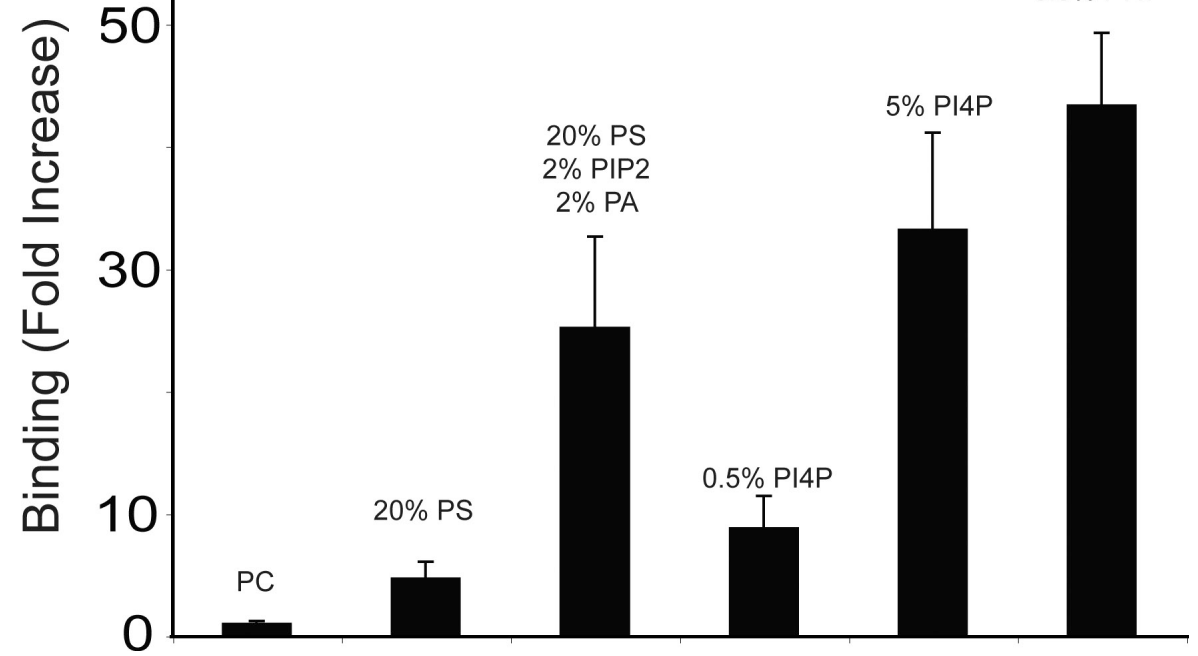

Figure 3. PIP5Ks interact with anionic phospholipids. (a) Purified GST or GST fusion proteins of PIP5K- $\alpha$ and $-\gamma 87$ were incubated with immobilized phospholipids and detected by immunoblotting using anti-GST antibodies. Chol, cholesterol; SM, sphingomyelin; TAG, triacylglycerol; $\mathrm{PE}$, phosphatidylethanolamine; $P G$, phosphatidylglycerol; $C L$, cardiolipin. Results are representative of three independent experiments. (b) Binding of PIP5K- $\alpha$ to lipidcoated beads. GFP-PIP5K- $\alpha$, partially purified from Hela cell extracts, was added to C18 Nucleosil beads coated with PC alone (i), 20\% PS + $80 \%$ PC (ii), 20\% PS + 2\% PA + 2\% PI4, $5 \mathrm{P}_{2}+$ $76 \% \mathrm{PC}$ (iii), $0.5 \% \mathrm{PI} 4 \mathrm{P}+99.5 \% \mathrm{PC}$ (iv), $5 \%$ $\mathrm{PI} 4 \mathrm{P}+95 \% \mathrm{PC}(\mathrm{v})$, or $0.5 \% \mathrm{PI} 4 \mathrm{P}+20 \% \mathrm{PS}+$ $2 \% \mathrm{PA}+2 \% \mathrm{PI} 4,5 \mathrm{P}_{2}+75.5 \% \mathrm{PC}$ (vi). Binding was assessed measuring the green fluorescence associated with the beads. Data are expressed relative to PC-only beads and are means \pm SEM of three separate experiments. surface charge of the membrane may contribute to the partitioning of the PIP5K isoforms. Accordingly, we found that all of the PIP5K isoforms colocalized with a recently described probe that identifies the most negatively charged membranes in the cell. As shown in Fig. 4 a, PIP5K- $\alpha,-\beta$, and both the $-\gamma 87$ and $-\gamma 90$ isoforms overlap extensively with arginine and prenylated-tagged RFP (R-Pre), which is a polycationic prenylated biosensor of surface charge (Yeung et al., 2006).

These results led us to investigate the possibility that the PIP5Ks function as coincidence detectors recognizing both PI4P and negatively charged membrane surfaces. To differentiate the contributions of PI4P and of electrostatic interactions to PIP5K targeting, we used the 5-phosphatase synaptojanin2. This enzyme converts $\mathrm{PI} 4,5 \mathrm{P}_{2}$, a primary contributor to the electronegativity of the inner leaflet of the plasma membrane, to PI4P (McPherson et al., 1996). Cells were cotransfected with a membrane-targeted form of the 5-phosphatase domain of synaptojanin2 (Sj2-CaaX; Malecz et al., 2000) and the $\mathrm{PH}$ domain of PLC- $\delta$ (PH-PLC- $\delta$ ). As illustrated in Fig. 4 b, the phosphatase reduced the $\mathrm{PI} 4,5 \mathrm{P}_{2}$ content of the membrane, releasing PH-PLC- $\delta$ to the cytosol. Under the same conditions, the $\mathrm{PH}$ domain of the yeast oxysterol-binding protein homologue 2, Osh2, shown previously to bind primarily to PI4P and to a lesser extent to PI4,5 $\mathrm{P}_{2}$ (Roy and Levine, 2004), remained associated with the membrane (Yeung et al., 2006). These findings imply that PI4P persisted in the inner face of the membrane and, in fact, that its concentration is likely to have increased as $\mathrm{PI} 4,5 \mathrm{P}_{2}$ was dephosphorylated in the 5 position.
We then proceeded to test the effects of $\mathrm{Sj} 2-\mathrm{CaaX}$ on the localization of the PIP5K isoforms. The phosphatase produced only a modest reduction in the association of PIP5K- $\beta$ to the membrane (Fig. 4 c). We reasoned that this small effect was attributable to the ability of catalytically active PIP5K- $\beta$ to counteract the disappearance of $\mathrm{PI} 4,5 \mathrm{P}_{2}$, antagonizing the effect of the phosphatase by resynthesizing the phosphoinositide. Indeed, we found that when cotransfected with $\mathrm{Sj} 2-\mathrm{CaaX}$, catalytically active PIP5K- $\beta$ minimized the release of PH-PLC- $\delta$ to the cytosol (unpublished data). To circumvent this problem, a catalytically inactive form of PIP5K- $\beta$ (PIP5K- $\beta-$ K179M) was cotransfected with $\mathrm{Sj} 2-\mathrm{CaaX}$. In this instance, $\mathrm{PH}-\mathrm{PLC}-\delta$ was effectively released from the plasmalemma. More importantly, the loss of $\mathrm{PI} 4,5 \mathrm{P}_{2}$ and concomitant reduction in the surface charge were accompanied by release of PIP5K- $\beta-\mathrm{K} 179 \mathrm{M}$ from the membrane (Fig. 4, b and c). Thus, the presence of PI4P is not sufficient to maintain the kinase at the plasma membrane, and $\mathrm{PI} 4,5 \mathrm{P}_{2}$, or more likely, the charge it confers to the membrane, is required for optimal targeting.

Additional experiments were performed to ensure that the wild-type kinases, and not just the catalytically inactive forms of PIP5K, also require the charge associated with $\mathrm{PI} 4,5 \mathrm{P}_{2}$ for targeting to the plasmalemma. To this end, production of polyphosphoinositides was terminated by depleting the cells of ATP. As shown in Fig. 5 a (insets), simultaneous inhibition of glycolysis and mitochondrial respiration induced depletion of $\mathrm{PI} 4,5 \mathrm{P}_{2}$, which was validated by the displacement of PH-PLC- $\delta$ from the membrane. In parallel, PIP5K- $\alpha,-\beta$, and both $-\gamma 87$ and 
Figure 4. PIP5Ks interact with anionic phospholipids. (a) Colocalization of PIP5K isoforms with the negative surface charge marker, R-Pre. (b) RAW cells were cotransfected with a kinase-deficient GFP-PIP5K- $\beta$ and RFP-PHPLC- $\delta$ without or with a construct encoding the 5-phosphatase domain of synaptojanin2 targeted to the membrane by attachment of a C-terminal CaaX box (Sj2-CaaX). (c) Quantification of the membrane association of GFP-PIP5K- $\beta$, kinasedeficient GFP-PIP5K- $\beta$, and RFP-PH-PLC- $\delta$ in the presence or absence of $\mathrm{Sj} 2$-CaaX. Data are means $\pm \operatorname{SEM}(n \geq 25) ;{ }^{*}, \mathrm{P}<0.001$ (d) The amino acid sequence of wild-type (WT) PIP5K- $\alpha$ from residues 410-464. The residues replaced in mutants $A$ and $B$ are highlighted in bold type with asterisks. (e) RAW cells were transiently cotransfected with GFP chimeras of with either wild-type PIP5K- $\alpha$ (left), mutant $A$ (middle), or mutant B and PM-RFP (right). The distribution of the fluorescent proteins was analyzed by spinning-disc confocal microscopy, and representative images acquired near the middle of the cell are illustrated. Bars, $3 \mu \mathrm{m}$.
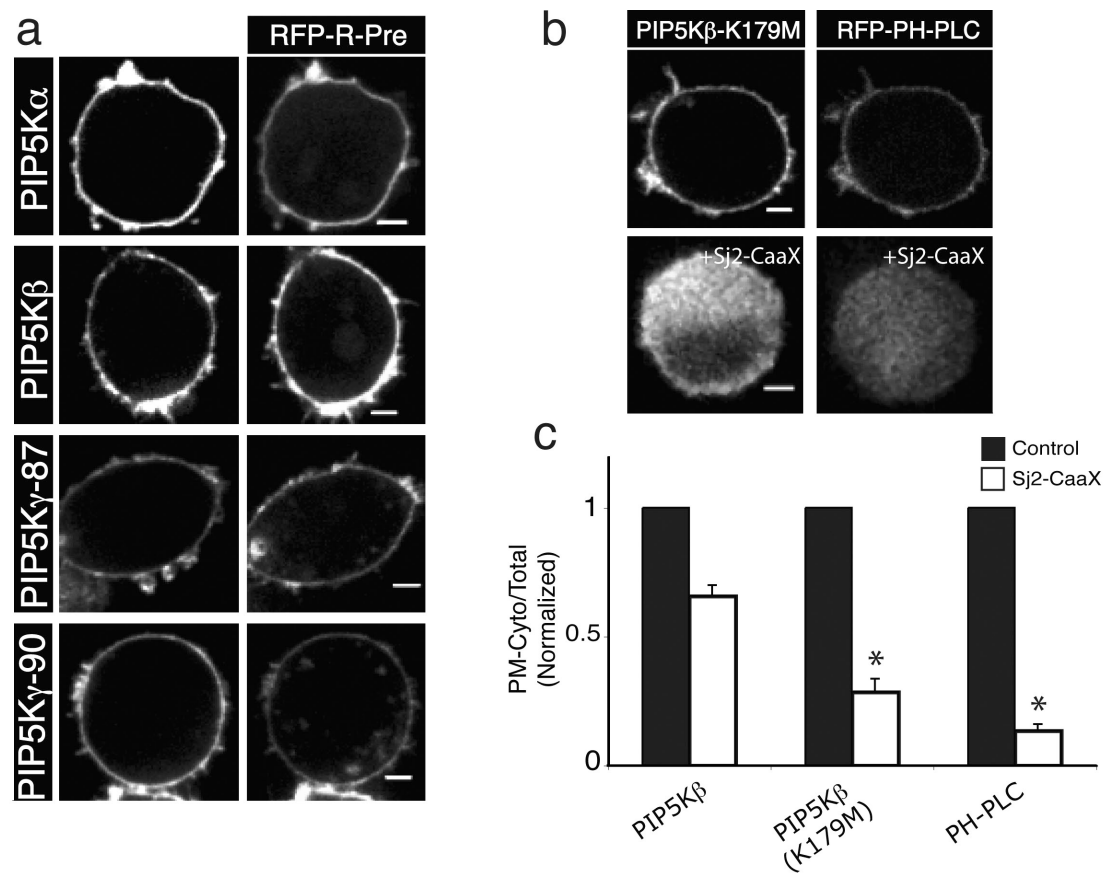

d WT 410-RFVKKLEHSWKALVHDGDTVSVHRPGFYAERFQRFMCNTVFKKIPLKPSPSKKFR-464 * * * * *

mut A 410-DFVDDLEHSWDALVHDGDTVSVHRPGFYAERFQRFMCNTVFKKIPLKPSPSKKFR-464 mut B 410-RFVKKLEHSWKALVHDGDTVSVHRPGFYAERFORFMCNTV******** mut B 410-RFVKKLEHSWKALVHDGDTVSVHRPGFYAERFQRFMCNTVFDDIPLDPSPSDDFD-464
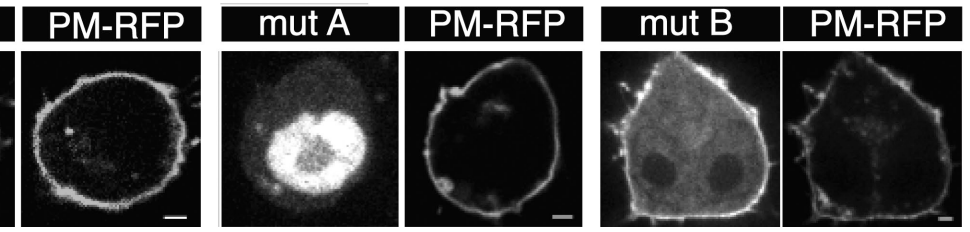

$-\gamma 90$ isoforms were largely released from the plasma membrane (Fig. 5, a and c).

An alternative way of reducing the negative charge of the inner leaflet of the plasma membrane is through an increase in cytosolic free calcium. The increase in calcium effectively decreases the surface charge by at least three synergistic mechanisms: (1) the cation directly shields the negative charges, (2) it activates the phosphoinositide-specific PLC, and (3) it promotes the scrambling of PS from the inner to the outer monolayer. When calcium was elevated in RAW cells by addition of the ionophore ionomycin, activation of PLC was evident by the release of PH-PLC- $\delta$ from the membrane (Fig. 5 b, insets). The appearance of PS on the outer surface and the concomitant decrease in the charge of the inner surface of the plasma membrane were also validated using annexin V and R-Pre, respectively (unpublished data). As in the case of ATP depletion, reduction of the surface charge by elevating calcium induced a marked redistribution of all of the PIP5K isoforms (Fig. 5, b and c).

\section{Neutralization of surface charge displaces PIP5K}

While lowering the membrane surface charge, all of the preceding manipulations also deplete intracellular $\mathrm{PI} 4,5 \mathrm{P}_{2}$. Thus, it is difficult to ascertain whether the effects on the kinases are caused by elimination of this phosphoinositide. We therefore sought means of altering the charge that do not entail hydrolysis of PI4,5 $\mathrm{P}_{2}$. It was previously documented that treating cells with dibucaine induces scrambling of PS from the inner to the outer leaflet of the plasma membrane (Yeung et al., 2006). Because PS constitutes $\geq 20$ mol\% of the lipids of the inner leaflet, such scrambling is anticipated to depress the membrane internal surface charge. Moreover, as a membrane-permeant cationic amphiphile, dibucaine inserts in the membrane and alters its net charge (Yeung et al., 2006). These combined effects rapidly reduce the surface charge of the plasma membrane, as indicated by the displacement of the R-Pre probe (Fig. S4 a). Of note, although the plasmalemmal charge was reduced, the PH-PLC- $\delta$ reporter remained associated with the plasma membrane, demonstrating that $\mathrm{PI} 4,5 \mathrm{P}_{2}$ was still present (Fig. S4, a and c). In parallel, all of the PIP5K isoforms were released from the plasma membrane (Fig. S4 c). Similar results were obtained by treating cells with squalamine, an aminosterol that, like dibucaine, can insert into membranes, contributing two positive charges per molecule that depress the anionic surface charge of the membrane (Yeung et al., 2006). Within minutes of adding squalamine, the PIP5Ks relocalized from the membrane to the cytoplasm (Fig. S4 b), whereas the PH-PLC- $\delta$ reporter remained associated with the plasma membrane (Fig. S4 a). 
a
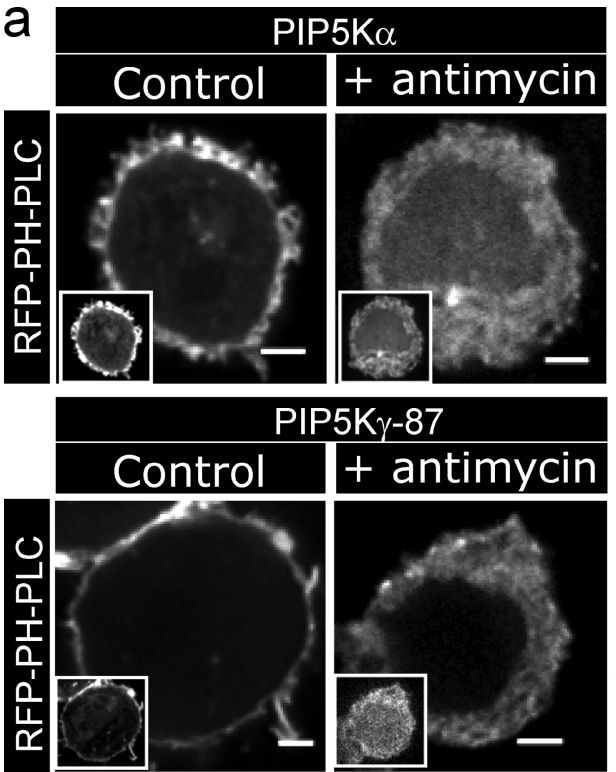

b
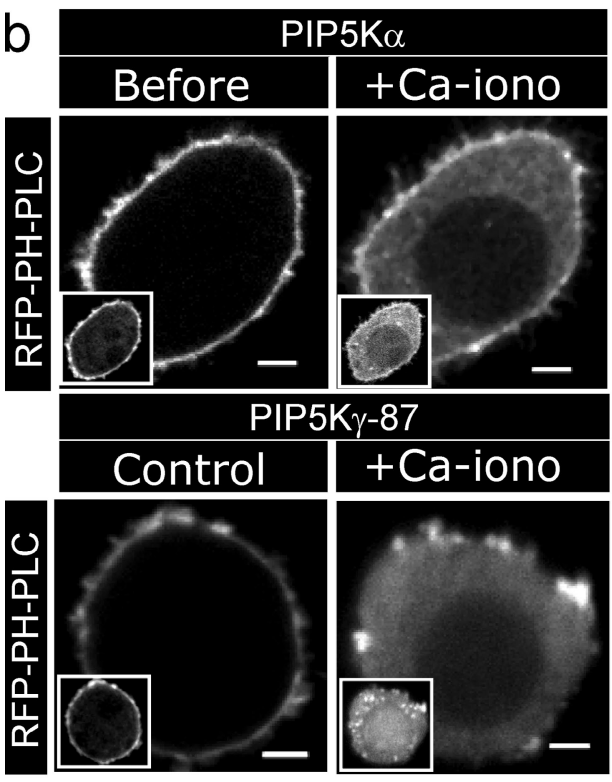

C

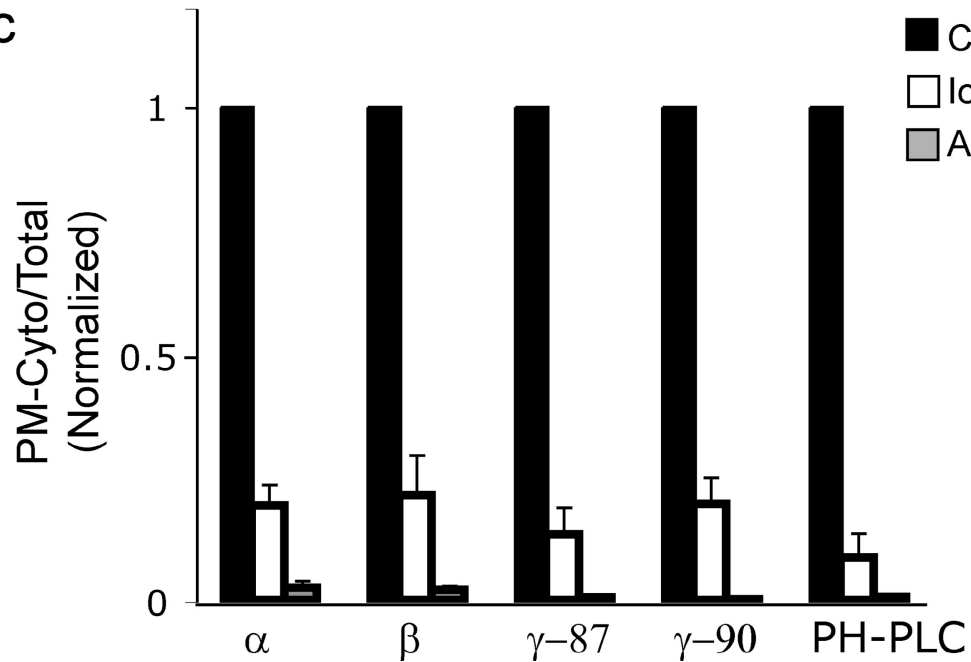

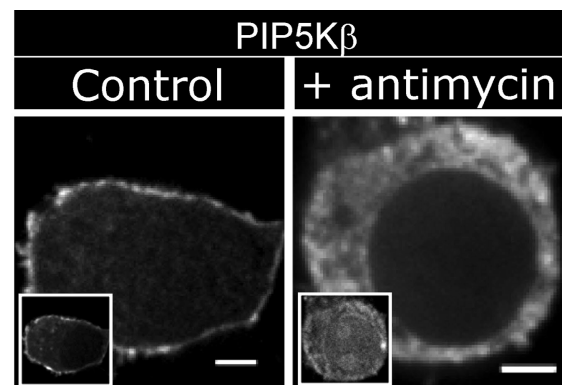
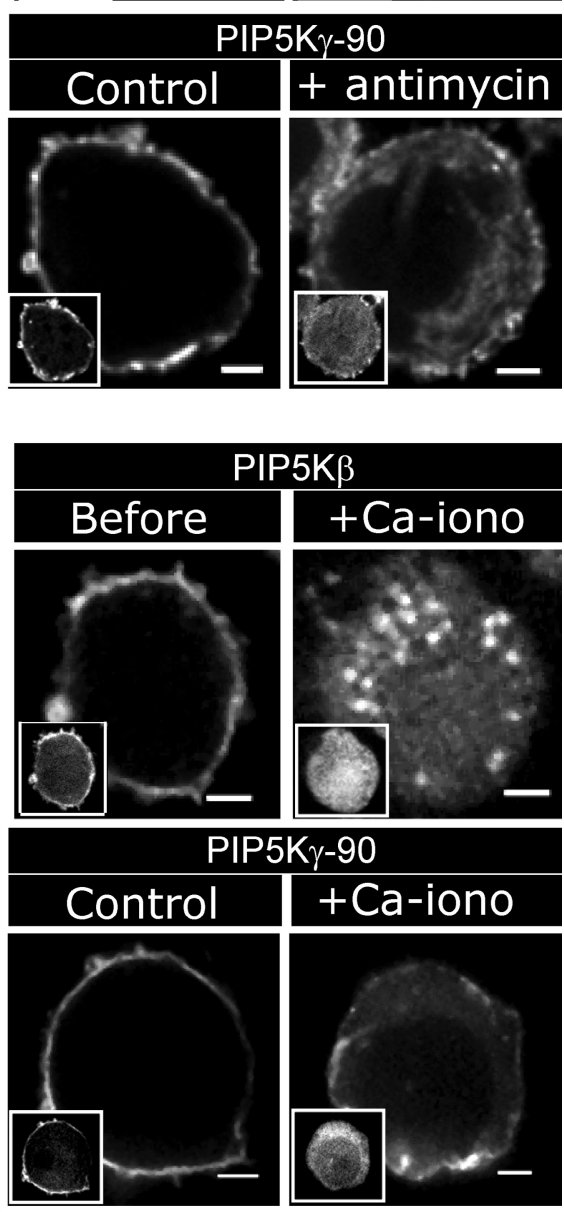

Control

$\square$ lonomycin

Antimycin
Figure 5. The localization of PIP5Ks is altered by depressing the surface charge. ( $a$ and $b$ ) Cells were cotransfected with the specified GFP-PIP5K isoform and RFP-PH-PLC- $\delta$ (insets). Images were acquired before and after incubation with either 2-deoxy-glucose plus antimycin for $40 \mathrm{~min}$ (a) or with $10 \mu \mathrm{M}$ ionomycin for $5 \mathrm{~min}$ (b). (c) Quantification of the membrane association of GFP-PIP5K isoforms and of PH-PLC- $\delta$ before and after the treatments described in $a$ and $b$. Data are means \pm SEM $(n \geq 30)$. Bars, $3 \mu \mathrm{m}$. 
These results imply that changing the surface charge suffices to release PIP5K from the membranes, regardless of the phosphoinositide content.

\section{The positive electrostatic potential of PIP5K is required for membrane association}

The preceding observations suggested that an electrostatic interaction contributes to PIP5K recruitment to the membrane. If this is the case, the association should be impaired not only by altering the charge of the membrane but also when the surface electrostatic potential of the protein is reduced. To assess this experimentally, we generated two mutant versions of PIP5K- $\alpha$ in which cationic residues were replaced to alter the charge of the region of protein predicted to associate with the membrane (Fig. 4 d). The distribution of GFP-tagged forms of these mutants was compared with that of the plasmalemmal probe PM-RFP, and representative results are illustrated in Fig. 4 e. Mutant A (PIP5K- $\alpha$ [R410D, K413D, K414D, K420D]) failed to associate with the membrane and instead was found in the cytosol and concentrated in the nucleus. Although mutant B (PIP5K- $\alpha$ [K451D, K452D, K456D, K461D, K462D, K464R]) was still detectable at the membrane, the association was much weaker. Image integration experiments showed that although $51.7 \pm 6 \%$ of the wild-type enzyme is membrane bound, only $8.4 \pm 2.8 \%$ (mean $\pm \mathrm{SD}$ ) of mutant $\mathrm{B}$ associates.

PIP5Ks are released from

forming phagosomes

It was shown previously that the lipid composition of the membrane changes during the course of phagosome formation and maturation (Araki et al., 1996; Botelho et al., 2000). These changes are accompanied by a considerable diminution in the surface charge of sealed phagosomes compared with the unengaged (bulk) plasma membrane (Yeung et al., 2006). Based on our preceding results, we anticipated that the association of PIP5K with the phagosomal membrane would be similarly altered. This prediction was tested experimentally using time-lapse microscopy to follow the localization of GFP-tagged forms of the kinases during the course of phagocytosis. As a reference, we used the PM-RFP probe to estimate the dilution of plasmalemmal components caused by exocytic insertion of endomembranes during and immediately after phagosome formation. As seen in Fig. 6 ( $\mathrm{a}$ and $\mathrm{b}$ ) and Video 1, the kinases were almost undetectable on the phagosomal membrane $180 \mathrm{~s}$ after initiation of phagocytosis. This depletion, which was observed for all of the isoforms tested, was not caused by wholesale membrane remodeling as a result of maturation because the PM-RFP probe was present in the sealed phagosomes at a density that was only slightly lower than that of the bulk plasmalemma (Fig. 6 b). These results indicate that the PIP5Ks dissociate from the membrane as the surface charge drops. Indeed, cotransfection experiments showed that the time course of dissociation of the kinases and of the R-Pre surface charge probe are virtually identical (Videos 1 and 2).

Earlier studies attributed the change in surface charge observed during phagocytosis primarily to the hydrolysis of $\mathrm{PI} 4,5 \mathrm{P}_{2}$ by PLC. Because PLC- $\gamma$ activation during phagocytosis requires
PI3,4,5 $\mathrm{P}_{3}$, we anticipated that inhibition of PI 3-kinase would affect the behavior of PIP5K. This prediction was tested in the experiments illustrated in Fig. 6 c. As described previously (Araki et al., 1996), in cells treated with wortmannin, pseudopods extend around the opsonized particle, but phagocytosis is arrested at an intermediate stage. Under these conditions, $\mathrm{PI} 4,5 \mathrm{P}_{2}$ is no longer hydrolyzed from the base of the phagocytic cup (Fig. $6 \mathrm{c}$, top), and the surface charge remains unaltered (Fig. $6 \mathrm{c}$, middle). Importantly, PIP5K remains associated with the forming phagosome. This observation furthers the correlation between the surface charge and the association of PIP5K with the plasma membrane.

\section{Prevention of PIP5K detachment} inhibits phagocytosis

Previous studies have suggested that disappearance of $\mathrm{PI} 4,5 \mathrm{P}_{2}$ from forming phagosomes is required for termination of actin polymerization, which was in turn proposed to be essential for phagosomal sealing and scission (Scott et al., 2005). Our aforementioned results suggest that an electrostatically induced detachment of PIP5K may contribute to the disappearance of PI4,5 $\mathrm{P}_{2}$ by locally terminating its synthesis. To test this notion, we used a form of PIP5K that could be recruited to the membrane in a manner that did not depend on charge interactions. Specifically, we expressed a chimeric construct consisting of the cDNA encoding the full-length 87-kD splice variant of PIP5K- $\gamma$ fused to the FK506-binding protein (FKBP) and tagged with YFP (YFP-FKBP-5K). Unlike the unmodified PIP5K- $\gamma 90$ and PIP5K- $\gamma 87$, this chimeric construct is partly cytosolic (Fig. 7 b). Attachment of the FKBP moiety to the $\mathrm{N}$ terminus of PIP5K- $\gamma 87$ seemingly impairs normal recruitment of the kinase to the membrane, possibly by preventing formation of homodimers or other protein interactions. However, as illustrated diagrammatically in Fig. 7 a, YFP-FKBP-5K can be effectively tethered to the membrane by coexpression of a membrane-targeted form of the rapamycin-binding domain of mTOR (FK506 rapamycin-binding domain [FRB]) followed by addition of rapamycin to induce heterodimerization of the two constructs (Inoue et al., 2005). In the absence of rapamycin, the membraneassociated fraction of YFP-FKBP-5K is displaced from the phagosomal cup in the course of particle ingestion (Fig. 7 b). Under these conditions, the macrophages can eliminate $\mathrm{PI} 4,5 \mathrm{P}_{2}$ from nascent phagosomes and effectively complete particle engulfment (Fig. 7 b). As shown in Fig. 7 c, in the absence of the cross-linker, the phagocytic efficiency of cells expressing YFP-FKBP-5K is only marginally lower than that of cells expressing FRB and YFP only. Upon addition of rapamycin, most of the YFP-FKBP-5K is retained on the plasma membrane by association with FRB and is not released when particles interact with the macrophages (Fig. 7 b). Importantly, the retention of excess PIP5K antagonizes the disappearance of $\mathrm{PI}, 5 \mathrm{P}_{2}$, which remains at the base of the phagosome, as shown by the persistent association of PH-PLC- $\delta$ (Fig. 7 b). As a consequence, actin fails to dissociate from the base of the phagocytic cup, and phagosome closure is impaired. The phagocytic index is markedly reduced when YFP-FKBP-5K is recruited to the plasma membrane by rapamycin (Fig. $7 \mathrm{c}$ ). This effect was not caused by rapamycin itself because the crosslinker produced no inhibition when added to cells expressing 
a
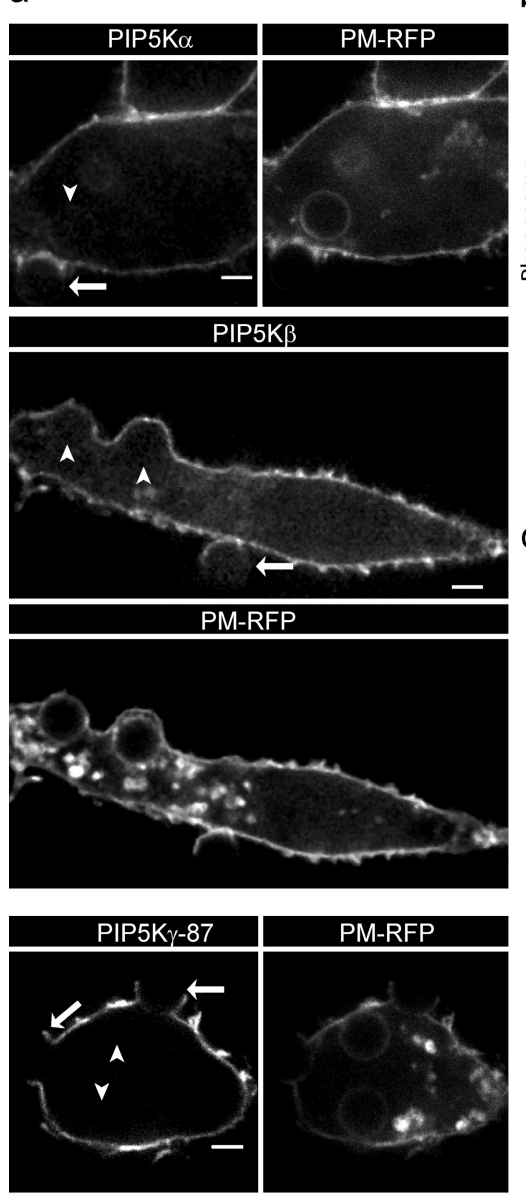

PIP5K $y-90$

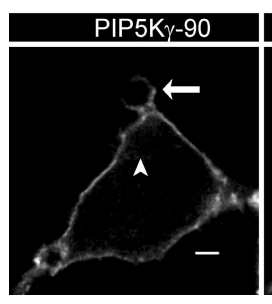

PM-RFP

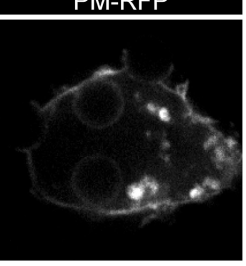

PM-RFP

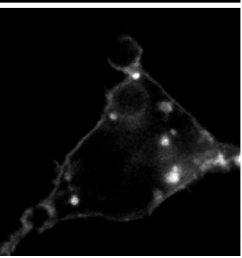

b

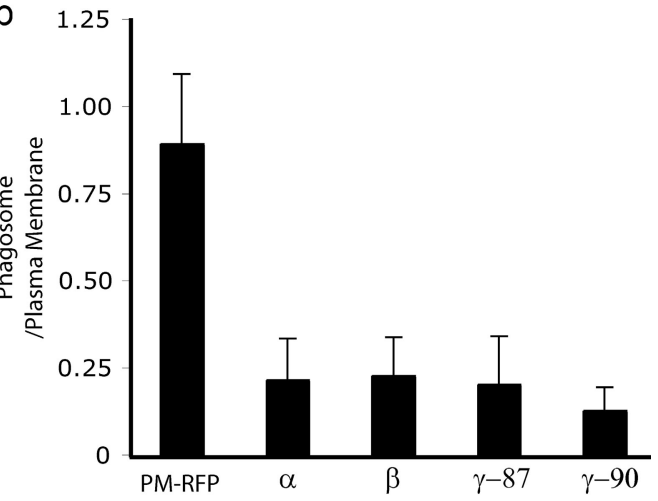

\section{C}
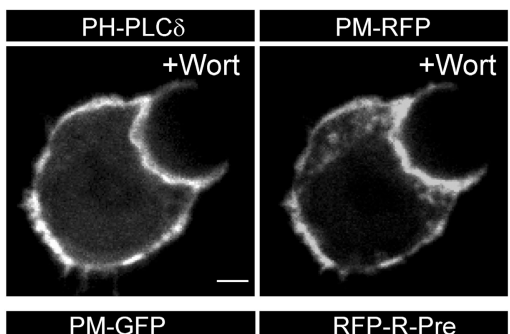

PM-GFP
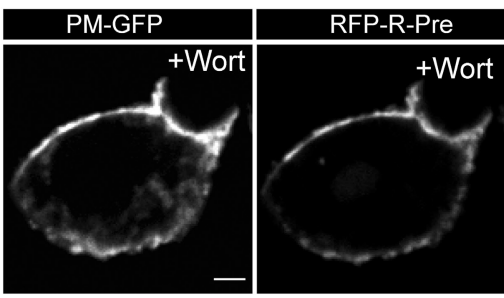

PIP5K $\gamma-87$

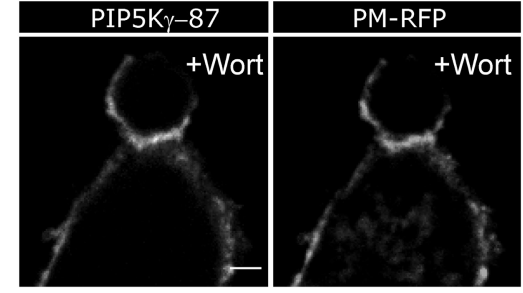

only YFP and FRB (Fig. 7 c). Together with our previous results, these observations suggest that the timely removal of PI4,5 $\mathrm{P}_{2}$ and depolymerization of actin from the base of the cup are required for the completion of phagocytosis.

\section{Discussion}

\section{Structural insights into the targeting of} type I PIPSKs

PIP5K isoforms have been detected in the nucleus (Payrastre et al., 1992; Boronenkov et al., 1998), focal adhesions (Ling et al., 2002), adherens junctions, and endosomes (Ling et al., 2007). Interaction with other proteins, such as Star-PAP, talin, and E-cadherin and clathrin adaptor (AP) complexes, are thought to target the kinases to these locations. In addition, recognition of its lipid substrate, PI4P, was proposed to direct PIP5K to the plasma membrane where the bulk of its activity occurs, as suggested by the subcellular distribution of its product, $\mathrm{PI} 4,5 \mathrm{P}_{2}$. The activation/substrate recognition loop is thought to target the class I

Figure 6. Redistribution of PIP5Ks during phagocytosis. (a) Macrophages were cotransfected with the specified GFP-PIP5K isoform and PM-RFP. Phagocytosis was initiated by exposure to lgG-opsonized beads, and the cells were imaged by spinning-disc microscopy. Arrowheads indicate sealed and internalized phagosomes, and arrows indicate forming phagosomes. The representative images shown were acquired $180 \mathrm{~s}$ after initiation of phagocytosis. (b) Quantification of the fluorescence intensity of the phagosomal membrane relative to the plasmalemma for each of the PIP5K isoforms and for PM-RFP. Data are means \pm SEM $(n \geq 30)$. (c) Cells were cotransfected with the indicated constructs and treated with $100 \mathrm{nM}$ wortmannin for $10 \mathrm{~min}$ before initiation of phagocytosis. The representative images shown were acquired $180 \mathrm{~s}$ after initiation of phagocytosis. Bars, $3 \mu \mathrm{m}$.
PIP5K to the membrane where it can interact with PI4P, which is a view supported by the generation of chimeric enzymes between class I and II kinases (Kunz et al., 2000). However, other proteins bearing PI4P-binding motifs are found predominantly in the Golgi complex where this phosphoinositide is thought to be abundant (Godi et al., 2004; Tóth et al., 2006). This paradoxical behavior suggests that, although necessary, PI4P may not be sufficient to direct and retain PIP5K at the plasma membrane. This prompted us to search for additional targeting determinants.

Analysis of the three-dimensional structure of the PIP5K isoforms provided useful insights. We took advantage of the reported three-dimensional structure of the type II- $\beta$ PIP4K to model the homologous type I enzymes. The type II- $\beta$ enzyme is known to form a dimer, and our homology modeling suggests that the type I enzymes may also exist as homodimers, exposing both catalytic sites on the same face of the protein. This relatively planar face of the protein also displays a notable accumulation of cationic residues, whereas anionic residues are preferentially displayed on the opposite side of the kinases. The resulting 
Figure 7. Sustained production of $\mathbf{P} 4,5 \mathrm{P}_{2}$ inhibits phagocytosis. (a) Schematic of the experimental protocol. The addition of rapamycin induces the heterodimerization of the YFP-FKBP-5K construct with a plasma membrane-targeted form of FRB. (b) Distribution of YFP-FKBP-5K and RFP-PH-PLC- $\delta$ before (-Rap) and 5 min after the addition of $10 \mu M$ rapamycin (+Rap). Arrowhead identifies sealed and internalized phagosomes, and the arrow highlights forming phagosomes. (c) Internalization and adherence of beads to macrophages was quantified in cells expressing YFP or YFP-FKBP-5K and plasma membrane-targeted FRB with or without the addition of rapamycin. Data are means \pm SEM of at least 500 beads per condition.

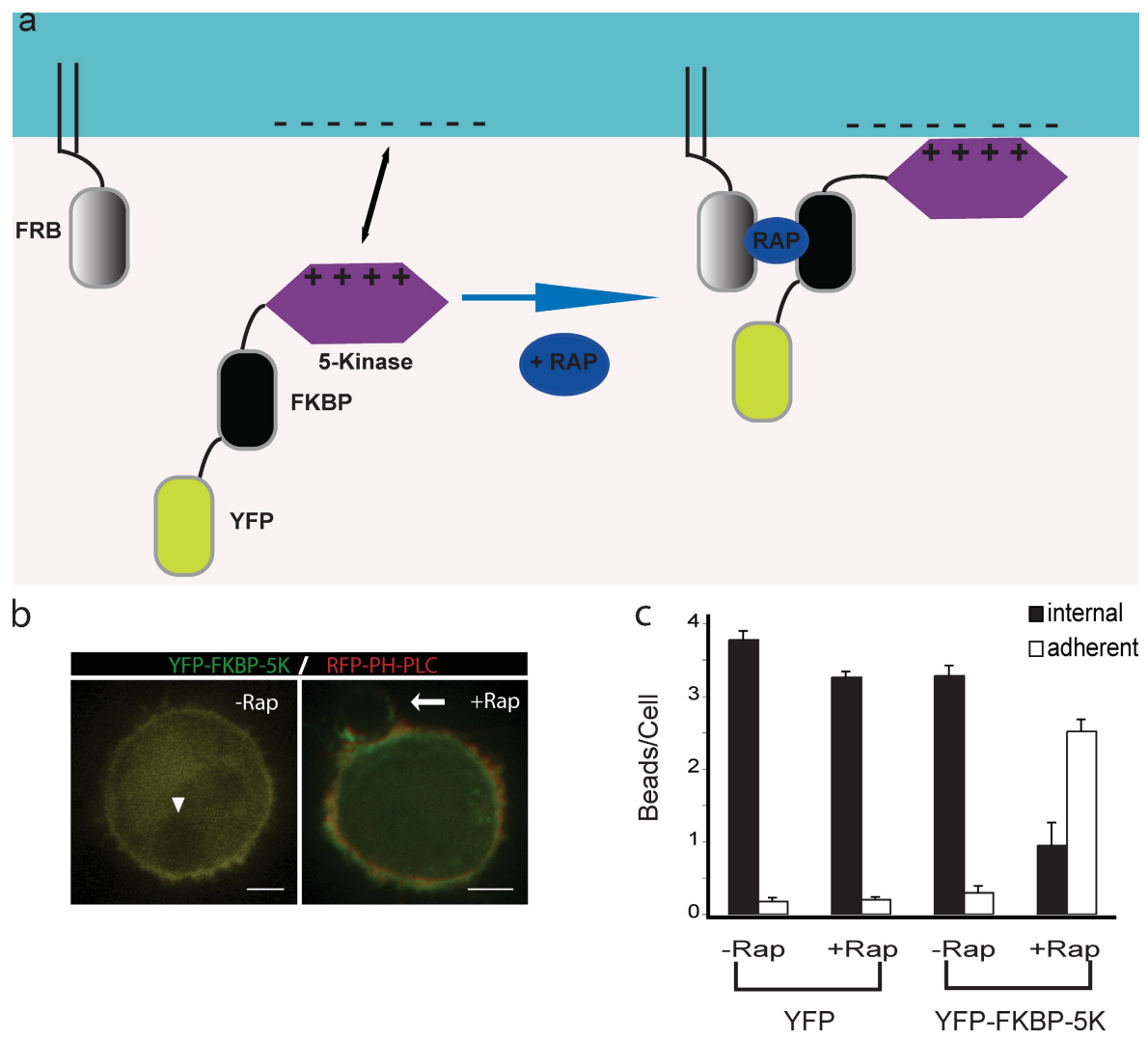

electrostatic dipole moment orients the protein, bringing the substrate recognition loop in close apposition with the anionic surface of the bilayer. Therefore, we believe that PIP5K functions as a coincidence detector, recognizing the plasma membrane by sensing PI4P in the context of a highly negative surface charge. Despite being endowed with PI4P, the Golgi complex may not be able to recruit significant amounts of PIP5K because its surface charge is considerably lower than that of the plasma membrane (Yeung et al., 2006; unpublished data). In support of this coincidence detection model, we found that PIP5Ks were displaced from the membrane by several procedures that decrease the surface charge without altering, or even despite increasing, its PI4P content.

\section{Role of electrostatic interactions in the regulation of PIPSKs}

The contribution of an electrostatic component to the recruitment of PIP5K to anionic membranes helps explain several published observations. First, in mixed micelle systems where the activity of the kinases was measured using a constant concentration of PI4P, the incorporation of negatively charged phospholipids was found to increase the rate of product formation $\sim 50$-fold (Jenkins et al., 1994). Furthermore, in vivo studies demonstrated that PLD-catalyzed production of phosphatidic acid can stimulate PIP5K activity (Divecha et al., 2000; Powner et al., 2005). Although phosphatidic acid could conceivably activate the enzyme directly by allosteric means, it is also possible that the additional anionic charge contributed by the phosphatidic acid increases the affinity of PIP5Ks for the membrane. It should be noted that phosphatidic acid has no effect on the catalytic activity of the type II kinases. Whether this is attributable to distinct allosteric effects or to differences in the electrostatic interactions dictated by differential distribution of charges on the surfaces of the type I and II enzymes remains to be defined.

In addition to anticipating the consequences of altering the surface charge of the membrane, the electrostatic model also predicts that changing the charge of the protein will affect its association with the bilayer. We validated this prediction by generating PIP5K $\alpha$ mutants with reduced cationic charge, which bound poorly or not at all to the plasma membrane (Fig. 4). These results are consistent with the observations of Suh et al. (2006), which demonstrated that a $\gamma 87$ mutant (RK445, 446EE) is also displaced from the plasma membrane. The charge of the protein can also be altered by physiological means. Phosphorylation of PIP5Ks could conceivably lower its affinity for the membrane, thereby reducing their catalytic effectiveness. In this regard, PIP5Ks have been shown to undergo autophosphorylation in vitro when in the presence of substrates (Itoh et al., 2000). Moreover, Src kinase activation can lead to phosphorylation of PIP5K $\alpha$, and this in turn displaces the kinase from the plasma membrane, leading to a depletion of $\mathrm{PI} 4,5 \mathrm{P}_{2}$ (Halstead et al., 2001). Conversely, dephosphorylation of PIP5K was reported to increase PI4,5 $\mathrm{P}_{2}$ synthesis. In HeLa cells, a pool of phosphorylated and ostensibly inactive PIP5K $\beta$ exists in the cytosol. Osmotic stress induces dephosphorylation of this pool followed by relocation of the kinases to the membrane, where they encounter their substrate and catalyze the formation of $\mathrm{PI} 4,5 \mathrm{P}_{2}$ (Yamamoto et al., 2006).

These observations suggest that PIP5K location may be controlled by an electrostatic switch. The concept of an electrostatic switch was introduced by McLaughlin and Aderem (1995) to explain the redistribution of myristoylated alanine-rich 
C kinase substrate that accompanies its phosphorylation and was later expanded to members of the Ras superfamily of GTPases (Bivona et al., 2006). Interestingly, Rho GTPases physically interact with PIP5Ks and stimulate their activity (Tolias et al., 1995; Weernink et al., 2004). Thus, the proposed electrostatic switch controlling the localization and activity of PIP5Ks may be toggled by altering the charge of the membrane, that of the kinase or its associated proteins.

The reciprocal relationship between the electrostatic dipole of the kinase and the charge of its product, $\mathrm{PI} 4,5 \mathrm{P}_{2}$, generates a positive feedback loop that confers unique, magnifying properties to their interaction. During phospholipase- or phosphataseinitiated $\mathrm{PI} 4,5 \mathrm{P}_{2}$ hydrolysis, the loss of charge will promote detachment of the kinase, accentuating the loss of PI4,5P 2 . Conversely, during resynthesis of $\mathrm{PI} 4,5 \mathrm{P}_{2}$ (e.g., during recovery from ATP depletion), more kinase will be recruited to the membrane progressively, as $\mathrm{PI} 4,5 \mathrm{P}_{2}$ is generated, accelerating the process (clearly, a minimum amount of the kinase must be present to initiate synthesis whether by interacting with proteins or attracted by PI4P or PI4, $5 \mathrm{P}_{2}$ delivered to the membrane by the secretory pathway). By accelerating the rates of $\mathrm{PI} 4,5 \mathrm{P}_{2}$ biosynthesis or disappearance, the reciprocal relationship between the kinase and the charge of its product generates a bistable electrostatic switch that magnifies $\mathrm{PI} 4,5 \mathrm{P}_{2}$ signaling at the membrane.

\section{PIP5K in phagocytosis}

PIP5K isoforms appear to play multiple roles in the phagocytic process. A recent study found that PIP5K- $\gamma$ influences the ability of phagocytic receptors to cluster after ligand engagement (Mao et al., 2009). This step precedes and is essential for the subsequent rearrangement of actin via WASP and Arp2/3, a complex event that requires PIP5K- $\alpha$. Actin undergoes an acute biphasic change during phagocytosis: it is initially polymerized at sites of particle contact and pseudopod protrusion and is then disassembled from the base of the phagocytic cup (Botelho et al., 2000). Disassembly is required for proper sealing and internalization of the target particles and also likely for fusion with endomembranes (O'Reilly et al., 2003; Scott et al., 2005). PI4,5 $\mathrm{P}_{2}$ appears to play a key role in both phases of actin remodelling: polymerization is accompanied by an increase in the local concentration of PI4,5 $\mathrm{P}_{2}$, whereas the phosphoinositide virtually disappears from the base of the phagosome as actin disassembles (Araki et al., 1996; Scott et al., 2005). These events are related in a causal manner because impairment of PI4,5P hydrolysis by directly (Scott et al., 2005) or indirectly inhibiting PLC- $\gamma$ (Araki et al., 1996) prevented the dissociation of actin from the phagocytic cup. Clearly, defining the means whereby PI4,5P $\mathrm{P}_{2}$ is eliminated from the forming phagosomes is key to understanding the phagocytic process.

Our results revealed that during the later stages of particle engulfment, PIP5Ks are acutely depleted from sites of phagocytosis. The depletion closely parallels the loss of $\mathrm{PI} 4,5 \mathrm{P}_{2}$, suggesting that the detachment of the kinase contributes to the disappearance of the phosphoinositide. This notion is supported by the observation that $\mathrm{PI} 4,5 \mathrm{P}_{2}$ persisted at the base of the phagosome when the kinase was forcibly retained by means of rapamycin-induced crosslinking. Moreover, we have repeatedly noted that overexpression of catalytically active PIP5K impairs phagocytosis, arresting the process at a stage where both actin and PI4,5 $\mathrm{P}_{2}$ are present at the base of the cup (unpublished data). Indeed, experiments like those in Fig. 6 were only feasible when modest amounts of the kinases were expressed, accounting for the comparatively dim fluorescence illustrated.

The detachment of PIP5K from nascent phagosomes coincides and is likely caused by the sharp drop in the surface charge of the membrane lining the cup. Direct measurements using R-Pre and related probes revealed a localized loss in negativity that was attributed to the hydrolysis of PI4,5 $\mathrm{P}_{2}$ (Yeung et al., 2006). As previously mentioned, the disappearance of $P I 4,5 P_{2}$ is caused primarily by activation of PLC- $\gamma$, and to a lesser extent, by conversion to $\mathrm{PI} 3,4,5 \mathrm{P}_{3}$. Formation of $\mathrm{PI} 3,4,5 \mathrm{P}_{3}$, which is required for optimal activation of PLC- $\gamma$, would be expected to make the surface charge more negative. However, the mole fraction of $\mathrm{PI} 4,5 \mathrm{P}_{2}$ that is converted to $\mathrm{PI} 3,4,5 \mathrm{P}_{3}$ is minute and greatly exceeded by the fraction converted to diacylglycerol. Moreover, the latter conversion entails a loss from the membrane of approximately four negative charges per molecule of $\mathrm{PI} 4,5 \mathrm{P}_{2}$ hydrolyzed compared with the gain of one negative charge when $\mathrm{PI} 3,4,5 \mathrm{P}_{3}$ is generated from $\mathrm{PI} 4,5 \mathrm{P}_{2}$. To date, the possible role of 5-phosphatases such as synaptojanin2 in the loss of PI4,5 $\mathrm{P}_{2}$ during phagocytosis has not been investigated. However, because synaptojanin 2 is known to be a Rac1 effector and loss of synaptojanin2 causes a decrease in clathrin-coated pits (Malecz et al., 2000), the contribution of synaptojanin 2 or other phosphatases in $\mathrm{PI} 4,5 \mathrm{P}_{2}$ loss during phagocytosis cannot be discounted.

In view of the preceding considerations, we envisage the following sequence of events: (a) upon engagement of phagocytic receptors, PIP5K is initially activated, possibly by the localized accumulation of phosphatidic acid (Corrotte et al., 2006) and/or by the stimulation of Rho family GTPases (Chong et al., 1994), resulting in elevated PI4,5 $\mathrm{P}_{2}$. Phosphatidylinositol 3-kinase is also activated by the receptors at an early stage. (b) $\mathrm{PI} 3,4,5 \mathrm{P}_{3^{-}}$ mediated recruitment and tyrosine phosphorylation of PLC- $\gamma$ activate the hydrolysis of $\mathrm{PI} 4,5 \mathrm{P}_{2}$, which in turn reduces the surface charge of the phagosomal membrane; and (c) PIP5Ks dissociate from the membrane in response to the drop in surface charge, thereby terminating $\mathrm{PI}, 5 \mathrm{P}_{2}$ synthesis and contributing to catastrophic depletion of this phosphoinositide. The resulting positive feedback loop accelerates the depolymerization of actin, favoring completion of phagocytosis. The results demonstrate that a feedback loop can accelerate the displacement of PIP5Ks from the membrane and can also reduce the membrane surface charge despite the presence of PI4P. These changes are diagrammatically illustrated in Fig. 8.

In summary, we described the regulation of PIP5K localization and, thus, activity by means of an electrostatic interaction with the membrane. The uniquely negative charge of the inner leaflet of the plasma membrane, in combination with the PI4P-binding ability of the activation loop, targets the kinases to the membrane. Changes in the charge of the kinase, such as by phosphorylation or in the surface charge of the membrane, potentially caused by lipid hydrolysis or divalent cation-mediated shielding, can modulate the affinity of the interaction. This electrostatic switch mechanism adds a new dimension to the regulation of PIP5K. 
Figure 8. Schematic representation of the changes in phospholipid content and surface charge during phagocytosis. Proposed changes in the content of $\mathrm{PI} 4,5 \mathrm{P}_{2}$ (blue), $\mathrm{PI} 3,4,5 \mathrm{P}_{3}$ (yellow), and surface charge of the membrane (red) during the various stages of phagocytosis shown chronologically from top to bottom.

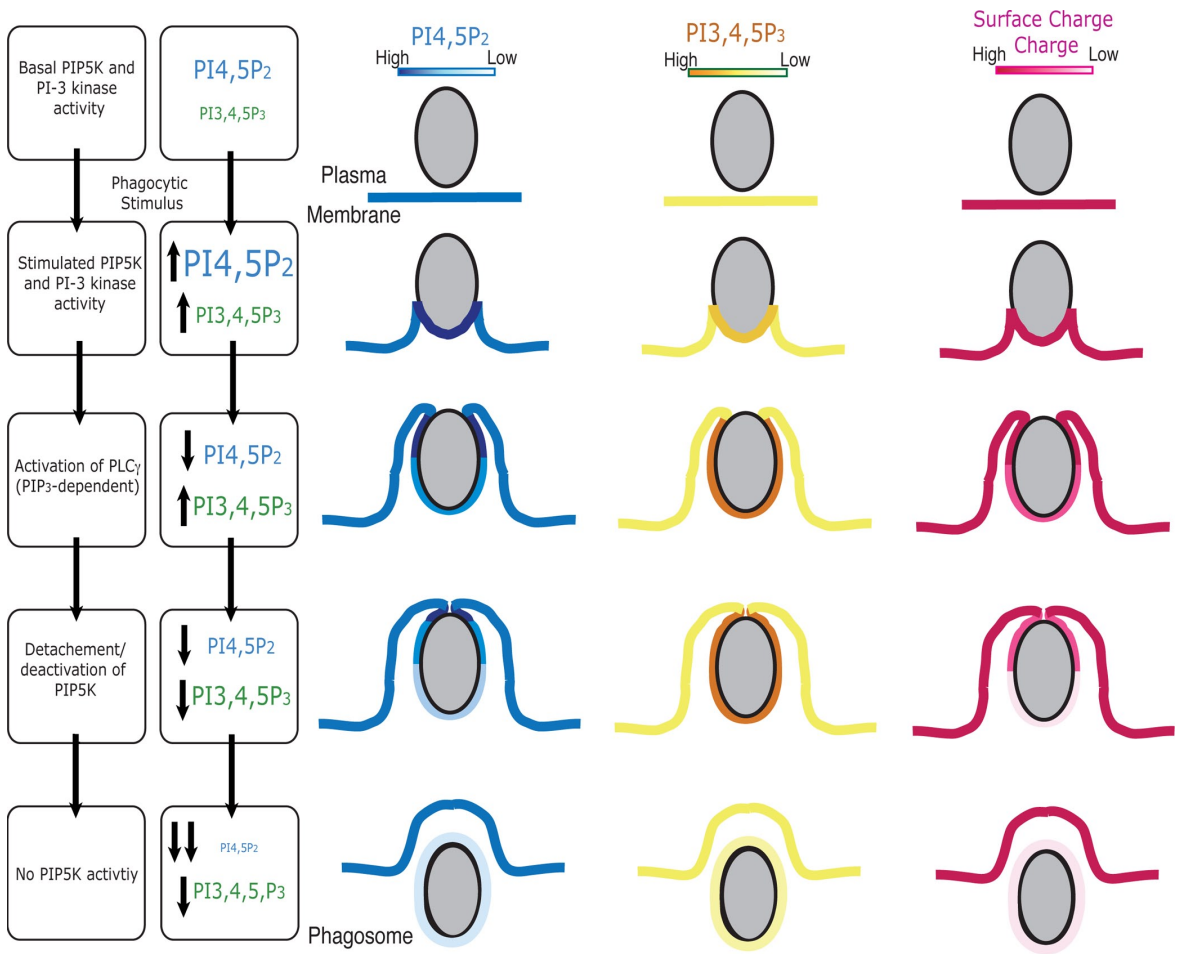

cloning site of the YFP-FKBP construct (Inove et al., 2005) using EcoRI and BamHI (New England Biolabs, Inc.).

\section{Pharmacological treatments}

Imaging experiments were performed in a synthetic medium consisting of $150 \mathrm{mM} \mathrm{NaCl}, 5 \mathrm{mM} \mathrm{KCl}, 1 \mathrm{mM} \mathrm{MgCl}$, 100 M EGTA, $2 \mathrm{mM} \mathrm{CaCl}$, and $20 \mathrm{mM}$ Hepes, $\mathrm{pH} 7.4$, with noted exceptions. ATP depletion experiments were performed by incubating cells for $30 \mathrm{~min}$ at $37^{\circ} \mathrm{C}$ in a similar medium lacking $\mathrm{CaCl}_{2}$ and glucose, additionally containing $200 \mathrm{nM}$ antimycin (Sigma-Aldrich) and $10 \mathrm{mM}$ 2-deoxy-D-glucose (Sigma-Aldrich). $1 \mathrm{mM}$ dibucaine (Sigma-Aldrich) was added to cells for $30 \mathrm{~min}$ in calcium-free medium. Cells were treated with $5 \mathrm{\mu g} / \mathrm{ml}$ squalamine in HPMl and monitored by time-lapse confocal microscopy.

\section{Fluorescence microscopy and image quantification}

Fluorescence images were acquired using spinning-disc confocal microscopy. The systems in use in our laboratory (Quorum) are based on a microscope (Axiovert 200M; Carl Zeiss, Inc.) with 63 or 100x objectives. The units are equipped with diode-pumped solid-state laser lines $(440,491$, 561, 638, and $655 \mathrm{~nm}$; Spectral Applied Research), motorized XY stage (Applied Scientific Instrumentation), and piezo focus drive. Images were acquired using back-thinned, electron-multiplied, or conventional cooled charge-coupled device cameras (Hamamatsu Photonics) driven by the Volocity software (version 4.1.1; PerkinElmer). Where indicated, the fluorescence intensity of defined regions of the cell was quantified using either ImageJ (National Institutes of Health) or Volocity. Regions of the cytosol or membrane were delineated in highly magnified images using the free-hand tool, and the mean fluorescence per pixel was determined. After background subtraction, the fluorescence of the cytosol was subtracted from that of the membrane, and the resulting value was divided by the mean total fluorescence to allow comparison between cells with varying levels of expression. Data from a minimum of 30 individual cells (or phagosomes) from at least three different experiments were analyzed for each condition.

\section{Protein purification and lipid binding}

Full-length open reading frames of PIP5K- $\alpha$ and $-\gamma 87$ in pGEX-4T3 vector were provided by H. Yin (University of Texas Southwestern Medical Center, Dallas, TX). Plasmids were transformed into Eschericia coli BL21 (DE3; $E M D$ ), and protein expression was induced by addition of $0.5 \mathrm{mM} \mathrm{IPTG}$ to exponential-phase bacteria. After $3 \mathrm{~h}$ at $30^{\circ} \mathrm{C}$, the bacteria were harvested and lysed with B-PER reagent (Thermo Fisher Scientific), and proteins were affinity purified using glutathione-Sepharose (GE Healthcare) and eluted with glutathione-containing buffers. 
Phospholipid-binding assays using lipid strips supplied by Echelon Biosciences, Inc. were performed essentially as described previously (Dowler et al., 2002). Blots were blocked with Tris-buffered saline containing 3\% (wt/vol) fatty acid-free bovine albumin. Purified GST fusion proteins were incubated at $4^{\circ} \mathrm{C}$ for $12 \mathrm{~h}$ with the immobilized phospholipids in Tris-buffered saline containing 3\% albumin. Blots were washed and incubated for $1 \mathrm{~h}$ at room temperature with anti-GST primary antibody $(1: 5,000)$ followed by an anti-mouse secondary antibody $(1: 5,000)$ coupled to HRP. After washing unbound antibodies, phospholipid binding was determined by ECL (Thermo Fisher Scientific).

The lipid selectivity of PIP5K- $\alpha$ was also tested using lipid-coated beads. For these experiments, 2 mg Nucleosil 120-3 C18 beads (3 pm; Richard Scientific) were resuspended in chloroform, and the specified lipid mixtures were added before drying under a stream of nitrogen. The bead lipid mixtures were resuspended in $75 \mathrm{mM} \mathrm{NaCl}, 2.5 \mathrm{mM} \mathrm{KCl}$, and $25 \mathrm{mM}$ Tris and sonicated until dispersed. Hela cells were cultured in 10-cm dishes and transfected using FuGene6 with $12 \mathrm{\mu g}$ cDNA encoding YFP-PIP5K$\alpha$. Before cell lysis, cells were incubated for $30 \mathrm{~min}$ in ATP depletion me$\operatorname{dium}(150 \mathrm{mM} \mathrm{NaCl}, 5 \mathrm{mM} \mathrm{KCl}, 1 \mathrm{mM} \mathrm{MgCl}, 2 \mathrm{mM} \mathrm{CaCl}$, and $20 \mathrm{mM}$ Hepes, pH 7.4, containing $200 \mathrm{nM}$ antimycin and $10 \mathrm{mM}$ deoxyglucose) to deplete polyphosphoinositides and release PIP5K- $\alpha$ from the membrane. Cells were scraped and collected in $300 \mu$ ITP depletion buffer. The volume of the suspension was brought up to $600 \mu \mathrm{l}$ with water, the cells were lysed by 20 strokes of a Dounce homogenizer, particulate material was sedimented using a table-top Airfuge (Beckman Coulter), and the supernatant was collected and used for lipid-binding experiments. Cell supernatants containing YFP-PIP5K- $\alpha$ were incubated with the lipid-coated beads for $5 \mathrm{~min}$ at $37^{\circ} \mathrm{C}$. Images were acquired using spinning-disc microscopy, and the mean fluorescence intensity of the beads was determined using Volocity.

\section{Phagocytosis}

RAW macrophages were grown on glass coverslips placed in 12-well plates with Dulbecco's minimum essential medium containing $5 \%$ fetal bovine serum. Before the experiments, the coverslips were transferred to Leiden chambers and bathed with HPMI. The chamber was placed in a thermostatted incubation holder mounted on the microscope stage. To initiate phagocytosis, 3.87- $\mu \mathrm{m}$ latex beads (Bang Beads) that had been freshly opsonized by incubation for $60 \mathrm{~min}$ with $2 \mathrm{mg} / \mathrm{ml}$ human lgG (Sigma-Aldrich) were added to the cells. When synchronization of phagocytosis was desired, the glass coverslips remained in their 12-well plate, and opsonized beads were added and rapidly sedimented onto the cells by centrifugation at 1,000 rpm for $1 \mathrm{~min}$ in a table-top centrifuge. Excess particles were removed by washing three times with PBS. Any remaining externally adherent beads were identified by immunostaining with a Cy5-conjugated anti-human lgG (Jackson ImmunoResearch Laboratories, Inc.) at $4^{\circ} \mathrm{C}$ for $1 \mathrm{~min}$. To determine the phagocytic index, phagocytosis was terminated at the specified times by placing the cells in the cold and fixing them with $4 \%$ paraformaldehyde.

\section{Homology modeling and analysis of the derived model}

Homology-modeling techniques were used to generate atomic models for the three dimensional structures of PIP5K- $\alpha$, PIP5K- $\beta$, and the $-\gamma 87$ and $-\gamma 90$ splice variants of PIP5K- $\gamma$. The amino acid sequences of these proteins were retrieved from Swiss-Prot (Boeckmann et al., 2003) entries (NCBI Protein database accession nos. Q99755, O14986, and O60331, respectively). A suitable structural template was identified by using Blast (Altschul et al., 1997) to search PDB (Berman et al., 2000) for entries exhibiting above-background sequence similarity with the three query sequences. The identified template, PIP4K type II- $\beta$ (PIP4K- $\beta$; chain A; PDB accession no. 1 boll), exhibits sequence identities of $32 \%, 33 \%$, and $32 \%$ with the partial sequences ( $90 \%$ for whole sequence) of PIP5K- $\alpha$, PIP5K- $\beta$, and PIP5K- $\gamma 90$, respectively. Next, the three query sequences were aligned onto the template sequence using the Needleman-Wunsch global alignment algorithm (Needleman and Wunsch, 1970). Gaps in the query protein positioned inside $\alpha$ helices and $\beta$ strands in the template structure were manually displaced toward residues in loop regions. Main chain atoms of the query proteins were assigned the spatial coordinates of backbone atoms corresponding to equivalent residues in the PIP4K- $\beta$ template. Side chain conformations were built onto the modeled backbones using the Metropolis Monte Carlo procedure implemented in the Modzinger software. Insertions and deletions in loop regions were modeled by searching a database of fragments from known protein structures in PDB for those that are compatible both with the target sequences and the template scaffold. Retrieved fragments were annealed with the template and subjected to energy minimization using classical molecular mechanics force fields from the AMBER package (Weiner et al., 1986). The loop-modeling procedure typically generates thousands of loop structures from which the structure with the lowest energy is selected for further analysis. The homodimer structures formed by the PIP5K isoforms were derived by superimposing the backbones of their modeled structures onto those of chains $A$ and $B$ in the PDB entry (accession no. 1bol). The superimpositions were followed by short energy minimizations to relieve close contacts.

The resulting models were validated using the Procheck software (Laskowski et al., 1993), which evaluates how the geometry and stereochemistry of a given atomic model compares with the corresponding parameters measured for known x-ray structure determined and deposited in PDB. As a measure of model quality, Procheck computes statistics on the backbone $(\varphi$ and $\phi)$ angles of the models, compares them to those expected for models of different accuracy ranges, and maps the values and expected ranges into the Ramachandran map.

The electrostatic potential on the protein surface and the dipole moment of the protein structures were computed for the modeled PIP5K isoforms as well as for the structure of PIP4K- $\beta$ template using the continuum solvation model embodied in the Poisson-Boltzmann method and the adaptive Poisson-Boltzmann solver implemented in the APBS software (using default parameters; Baker et al., 2001). Before running these calculations, hydrogen atoms were added to the protein structures, and partial charges were assigned using the pdb2qpr software (Dolinsky et al., 2007). Dipole moments of the PIP5K isoforms were calculated directly from the atomic models and the atom partial charges using Protein Dipole Moments Server (Felder et al., 2007). The electrostatic potentials on the protein surfaces and the dipole moments were illustrated using the VMD software (Humphrey et al., 1996).

\section{Online supplemental material}

Fig. S1 shows the Ramachandran plot of the four PI phosphate kinases. Fig. S2 shows sequence alignment of the PIP4K and PIP5K proteins and relevant sequence patterns. Fig. S3 shows position of catalytic and positively charged residues of the PIP4K and PIP5K proteins. Fig. S4 shows that localization of PIP5Ks is altered by a reduction in membrane surface charge. Video 1 shows distribution of PIP5K- $\gamma 87$ during phagocytosis. Video 2 shows distribution of R-Pre during phagocytosis. Online supplemental material is available at http://www.jcb.org/cgi/content/full/jcb.200909025/DC1.

This work was supported by Canadian Institutes of Health Research (CIHR; grant 7075). G.D. Fairn is the recipient of CIHR postdoctoral fellowship, and S. Grinstein is the current holder of the Pitblado Chair in Cell Biology.

Submitted: 3 September 2009

Accepted: 2 November 2009

\section{References}

Altschul, S.F., T.L. Madden, A.A. Schäffer, J. Zhang, Z. Zhang, W. Miller, and D.J. Lipman. 1997. Gapped BLAST and PSI-BLAST: a new generation of protein database search programs. Nucleic Acids Res. 25:3389-3402. doi:10.1093/nar/25.17.3389

Araki, N., M.T. Johnson, and J.A. Swanson. 1996. A role for phosphoinositide 3-kinase in the completion of macropinocytosis and phagocytosis by macrophages. J. Cell Biol. 135:1249-1260. doi:10.1083/jcb.135.5.1249

Baker, N.A., D. Sept, S. Joseph, M.J. Holst, and J.A. McCammon. 2001. Electrostatics of nanosystems: application to microtubules and the ribosome. Proc. Natl. Acad. Sci. USA. 98:10037-10041. doi:10.1073/pnas .181342398

Berman, H.M., J. Westbrook, Z. Feng, G. Gilliland, T.N. Bhat, H. Weissig, I.N. Shindyalov, and P.E. Bourne. 2000. The Protein Data Bank. Nucleic Acids Res. 28:235-242. doi:10.1093/nar/28.1.235

Bivona, T.G., S.E. Quatela, B.O. Bodemann, I.M. Ahearn, M.J. Soskis, A. Mor, J. Miura, H.H. Wiener, L. Wright, S.G. Saba, et al. 2006. PKC regulates a farnesyl-electrostatic switch on K-Ras that promotes its association with Bcl-XL on mitochondria and induces apoptosis. Mol. Cell. 21:481-493. doi:10.1016/j.molcel.2006.01.012

Boeckmann, B., A. Bairoch, R. Apweiler, M.C. Blatter, A. Estreicher, E. Gasteiger, M.J. Martin, K. Michoud, C. O'Donovan, I. Phan, et al. 2003. The SWISS-PROT protein knowledgebase and its supplement TrEMBL in 2003. Nucleic Acids Res. 31:365-370. doi:10.1093/nar/gkg095

Boronenkov, I.V., J.C. Loijens, M. Umeda, and R.A. Anderson. 1998. Phosphoinositide signaling pathways in nuclei are associated with nuclear speckles containing pre-mRNA processing factors. Mol. Biol. Cell. 9:3547-3560.

Botelho, R.J., M. Teruel, R. Dierckman, R. Anderson, A. Wells, J.D. York, T. Meyer, and S. Grinstein. 2000. Localized biphasic changes in 
phosphatidylinositol-4,5-bisphosphate at sites of phagocytosis. J. Cell Biol. 151:1353-1368. doi:10.1083/jcb.151.7.1353

Burden, L.M., V.D. Rao, D. Murray, R. Ghirlando, S.D. Doughman, R.A. Anderson, and J.H. Hurley. 1999. The flattened face of type II beta phosphatidylinositol phosphate kinase binds acidic phospholipid membranes. Biochemistry. 38:15141-15149. doi:10.1021/bi991571a

Chong, L.D., A. Traynor-Kaplan, G.M. Bokoch, and M.A. Schwartz. 1994. The small GTP-binding protein Rho regulates a phosphatidylinositol 4-phosphate 5-kinase in mammalian cells. Cell. 79:507-513. doi:10.1016/ 0092-8674(94)90259-3

Corrotte, M., S. Chasserot-Golaz, P. Huang, G. Du, N.T. Ktistakis, M.A. Frohman, N. Vitale, M.F. Bader, and N.J. Grant. 2006. Dynamics and function of phospholipase $\mathrm{D}$ and phosphatidic acid during phagocytosis. Traffic. 7:365-377. doi:10.1111/j.1600-0854.2006.00389.x

D’Angelo, G., M. Vicinanza, A. Di Campli, and M.A. De Matteis. 2008. The multiple roles of PtdIns(4)P — not just the precursor of PtdIns(4,5)P2. J. Cell Sci. 121:1955-1963. doi:10.1242/jcs.023630

Divecha, N., M. Roefs, J.R. Halstead, S. D'Andrea, M. Fernandez-Borga, L. Oomen, K.M. Saqib, M.J. Wakelam, and C. D'Santos. 2000. Interaction of the type Ialpha PIPkinase with phospholipase D: a role for the local generation of phosphatidylinositol 4, 5-bisphosphate in the regulation of PLD2 activity. EMBO J. 19:5440-5449. doi:10.1093/emboj/19.20.5440

Dolinsky, T.J., P. Czodrowski, H. Li, J.E. Nielsen, J.H. Jensen, G. Klebe, and N.A. Baker. 2007. PDB2PQR: expanding and upgrading automated preparation of biomolecular structures for molecular simulations. Nucleic Acids Res. 35:W522-W525. doi:10.1093/nar/gkm276

Dowler, S., G. Kular, and D.R. Alessi. 2002. Protein lipid overlay assay. Sci. STKE. 2002:pl6. doi:10.1126/stke.2002.129.pl6

Felder, C.E., J. Prilusky, I. Silman, and J.L. Sussman. 2007. A server and database for dipole moments of proteins. Nucleic Acids Res. 35:W512-W521. doi:10.1093/nar/gkm307

Godi, A., A. Di Campli, A. Konstantakopoulos, G. Di Tullio, D.R. Alessi, G.S. Kular, T. Daniele, P. Marra, J.M. Lucocq, and M.A. De Matteis. 2004. FAPPs control Golgi-to-cell-surface membrane traffic by binding to ARF and PtdIns(4)P. Nat. Cell Biol. 6:393-404. doi:10.1038/ncb1119

Halstead, J.R., M. Roefs, C.D. Ellson, S. D’Andrea, C. Chen, C.S. D’Santos, and N. Divecha. 2001. A novel pathway of cellular phosphatidylinositol(3,4,5)-trisphosphate synthesis is regulated by oxidative stress. Curr. Biol. 11:386-395. doi:10.1016/S0960-9822(01)00121-X

Hammond, G.R., G. Schiavo, and R.F. Irvine. 2009. Immunocytochemical techniques reveal multiple, distinct cellular pools of PtdIns4P and PtdIns(4,5)P(2). Biochem. J. 422:23-35. doi:10.1042/BJ20090428

Humphrey, W., A. Dalke, and K. Schulten. 1996. VMD: visual molecular dynamics. J. Mol. Graph. 14:33-38, 27-28.

Inoue, T., W.D. Heo, J.S. Grimley, T.J. Wandless, and T. Meyer. 2005. An inducible translocation strategy to rapidly activate and inhibit small GTPase signaling pathways. Nat. Methods. 2:415-418. doi:10.1038/ nmeth763

Itoh, T., H. Ishihara, Y. Shibasaki, Y. Oka, and T. Takenawa. 2000. Autophosphorylation of type I phosphatidylinositol phosphate kinase regulates its lipid kinase activity. J. Biol. Chem. 275:19389-19394. doi:10.1074/jbc.M000426200

Jarquin-Pardo, M., A. Fitzpatrick, F.J. Galiano, E.A. First, and J.N. Davis. 2007. Phosphatidic acid regulates the affinity of the murine phosphatidylinositol 4-phosphate 5-kinase-Ibeta for phosphatidylinositol-4-phosphate. J. Cell. Biochem. 100:112-128. doi:10.1002/jcb.21027

Jenkins, G.H., P.L. Fisette, and R.A. Anderson. 1994. Type I phosphatidylinositol 4-phosphate 5-kinase isoforms are specifically stimulated by phosphatidic acid. J. Biol. Chem. 269:11547-11554.

Kunz, J., M.P. Wilson, M. Kisseleva, J.H. Hurley, P.W. Majerus, and R.A. Anderson. 2000. The activation loop of phosphatidylinositol phosphate kinases determines signaling specificity. Mol. Cell. 5:1-11. doi:10.1016/S1097-2765(00)80398-6

Kunz, J., A. Fuelling, L. Kolbe, and R.A. Anderson. 2002. Stereo-specific substrate recognition by phosphatidylinositol phosphate kinases is swapped by changing a single amino acid residue. J. Biol. Chem. 277:5611-5619. doi:10.1074/jbc.M110775200

Laskowski, R.A., M.W. MacArthur, D.S. Moss, and J.M. Thornton. 1993. PROCHECK: a program to check the stereochemical quality of protein structures. J. Appl. Cryst. 26:283-291. doi:10.1107/S0021889892009944

Ling, K., R.L. Doughman, A.J. Firestone, M.W. Bunce, and R.A. Anderson. 2002. Type I gamma phosphatidylinositol phosphate kinase targets and regulates focal adhesions. Nature. 420:89-93. doi:10.1038/nature01082

Ling, K., S.F. Bairstow, C. Carbonara, D.A. Turbin, D.G. Huntsman, and R.A. Anderson. 2007. Type I $\gamma$ phosphatidylinositol phosphate kinase modulates adherens junction and E-cadherin trafficking via a direct interaction with $\mu 1$ B adaptin. J. Cell Biol. 176:343-353. doi:10.1083/jcb.200606023
Malecz, N., P.C. McCabe, C. Spaargaren, R. Qiu, Y. Chuang, and M. Symons. 2000. Synaptojanin 2, a novel Rac1 effector that regulates clathrin-mediated endocytosis. Curr. Biol. 10:1383-1386. doi:10.1016/S0960-9822(00)00778-8

Mao, Y.S., M. Yamaga, X. Zhu, Y. Wei, H.Q. Sun, J. Wang, M. Yun, Y. Wang, G. Di Paolo, M. Bennett, et al. 2009. Essential and unique roles of PIP5K- $\gamma$ and $-\alpha$ in Fc $\gamma$ receptor-mediated phagocytosis. J. Cell Biol. 184:281-296. doi:10.1083/jcb.200806121

McLaughlin, S., and A. Aderem. 1995. The myristoyl-electrostatic switch: a modulator of reversible protein-membrane interactions. Trends Biochem. Sci. 20:272-276. doi:10.1016/S0968-0004(00)89042-8

McPherson, P.S., E.P. Garcia, V.I. Slepnev, C. David, X. Zhang, D. Grabs, W.S. Sossin, R. Bauerfeind, Y. Nemoto, and P. De Camilli. 1996. A presynaptic inositol-5-phosphatase. Nature. 379:353-357. doi:10.1038/379353a0

Needleman, S.B., and C.D. Wunsch. 1970. A general method applicable to the search for similarities in the amino acid sequence of two proteins. J. Mol. Biol. 48:443-453. doi:10.1016/0022-2836(70)90057-4

O'Reilly, P.J., J.M. Hickman-Davis, I.C. Davis, and S. Matalon. 2003. Hyperoxia impairs antibacterial function of macrophages through effects on actin. Am. J. Respir. Cell Mol. Biol. 28:443-450. doi:10.1165/rcmb.2002-0153OC

Payrastre, B., M. Nievers, J. Boonstra, M. Breton, A.J. Verkleij, and P.M. Van Bergen en Henegouwen. 1992. A differential location of phosphoinositide kinases, diacylglycerol kinase, and phospholipase $\mathrm{C}$ in the nuclear matrix. J. Biol. Chem. 267:5078-5084.

Powner, D.J., R.M. Payne, T.R. Pettitt, M.L. Giudici, R.F. Irvine, and M.J. Wakelam. 2005. Phospholipase D2 stimulates integrin-mediated adhesion via phosphatidylinositol 4-phosphate 5-kinase Igamma b. J. Cell Sci. 118:2975-2986. doi:10.1242/jcs.02432

Rameh, L.E., K.F. Tolias, B.C. Duckworth, and L.C. Cantley. 1997. A new pathway for synthesis of phosphatidylinositol-4,5-bisphosphate. Nature. 390:192-196. doi:10.1038/36621

Rao, V.D., S. Misra, I.V. Boronenkov, R.A. Anderson, and J.H. Hurley. 1998. Structure of type IIbeta phosphatidylinositol phosphate kinase: a protein kinase fold flattened for interfacial phosphorylation. Cell. 94:829-839. doi:10.1016/S0092-8674(00)81741-9

Roy, A., and T.P. Levine. 2004. Multiple pools of phosphatidylinositol 4-phosphate detected using the pleckstrin homology domain of Osh2p. J. Biol. Chem. 279:44683-44689. doi:10.1074/jbc.M401583200

Scott, C.C., W. Dobson, R.J. Botelho, N. Coady-Osberg, P. Chavrier, D.A. Knecht, C. Heath, P. Stahl, and S. Grinstein. 2005. Phosphatidylinositol4,5-bisphosphate hydrolysis directs actin remodeling during phagocytosis. J. Cell Biol. 169:139-149. doi:10.1083/jcb.200412162

Stauffer, T.P., S. Ahn, and T. Meyer. 1998. Receptor-induced transient reduction in plasma membrane PtdIns(4,5)P2 concentration monitored in living cells. Curr. Biol. 8:343-346. doi:10.1016/S0960-9822(98)70135-6

Suh, B.C., T. Inoue, T. Meyer, and B. Hille. 2006. Rapid chemically induced changes of PtdIns(4,5)P2 gate KCNQ ion channels. Science. 314:14541457. doi: $10.1126 /$ science. 1131163

Teruel, M.N., T.A. Blanpied, K. Shen, G.J.Augustine, and T. Meyer. 1999. A versatile microporation technique for the transfection of cultured CNS neurons. J. Neurosci. Methods. 93:37-48. doi:10.1016/S0165-0270(99)00112-0

Tolias, K.F., L.C. Cantley, and C.L. Carpenter. 1995. Rho family GTPases bind to phosphoinositide kinases. J. Biol. Chem. 270:17656-17659. doi:10.1074/ jbc. 270.30 .17656

Tóth, B., A. Balla, H. Ma, Z.A. Knight, K.M. Shokat, and T. Balla. 2006. Phosphatidylinositol 4-kinase IIIbeta regulates the transport of ceramide between the endoplasmic reticulum and Golgi. J. Biol. Chem. 281:3636936377. doi:10.1074/jbc.M604935200

Várnai, P., and T. Balla. 1998. Visualization of phosphoinositides that bind pleckstrin homology domains: calcium- and agonist-induced dynamic changes and relationship to myo- $\left[{ }^{3} \mathrm{H}\right]$ inositol-labeled phosphoinositide pools. J. Cell Biol. 143:501-510. doi:10.1083/jcb.143.2.501

Weernink, P.A., K. Meletiadis, S. Hommeltenberg, M. Hinz, H. Ishihara, M. Schmidt, and K.H. Jakobs. 2004. Activation of type I phosphatidylinositol 4-phosphate 5-kinase isoforms by the Rho GTPases, RhoA, Rac1, and Cdc42. J. Biol. Chem. 279:7840-7849. doi:10.1074/jbc.M312737200

Weiner, S.J., P.A. Kollman, D.T. Nguyen, and D.A. Case. 1986. An all atom force field for simulations of proteins and nucleic acids. J. Comput. Chem. 7:230-252. doi:10.1002/jcc.540070216

Yamamoto, M., M.Z. Chen, Y.J. Wang, H.Q. Sun, Y. Wei, M. Martinez, and H.L. Yin. 2006. Hypertonic stress increases phosphatidylinositol 4,5-bisphosphate levels by activating PIP5KIbeta. J. Biol. Chem. 281:32630-32638. doi:10.1074/jbc.M605928200

Yeung, T., M. Terebiznik, L. Yu, J. Silvius, W.M. Abidi, M. Philips, T. Levine, A. Kapus, and S. Grinstein. 2006. Receptor activation alters inner surface potential during phagocytosis. Science. 313:347-351. doi:10.1126/ science. 1129551 

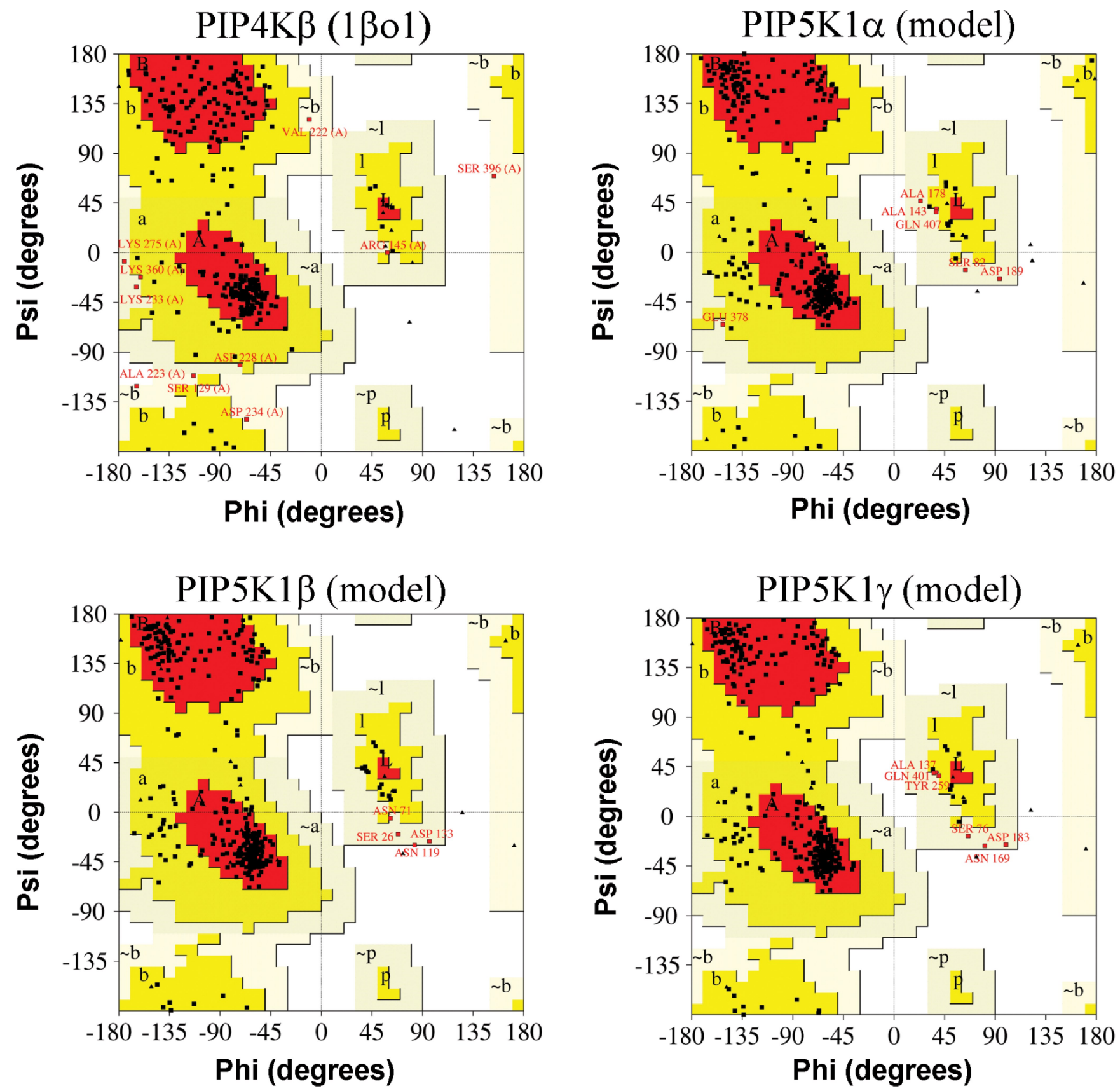

Figure S1. Ramachandran plot of the four PI phosphate kinases. PIP4K- $\beta$ (a), PIP5K- $\alpha$ (b), PIP5K- $\beta$ (c), and PIP5K- 990 (d) are shown. The plot was obtained using the Procheck software (Laskowski et al., 1993), which evaluates how the geometry and stereochemistry of a given atomic model compare with the corresponding parameters measured in known protein x-ray structures deposited in PDB. The most favored (red), additional allowed (yellow), generously allowed (light yellow), and disallowed regions (white) are indicated. Individual residues marked with $p$ (an allowed epsilon value) or I (an allowed left-handed helix) are also shown. The overall Procheck quality score (G factor), which combines backbone and side chain validation criteria, was found to be 0.02 , -0.42 , and -0.36 for PIP5K- $\alpha$, PIP5K- $\beta$, and PIP5K- $\gamma$, respectively. These values are within the range of good-quality crystal structures $(-0.8$ to $\sim 0.3)$ and are only marginally worse than the value computed for the structure of the template protein $(0.16)$. 
PI P 4 K

PI P $5 \mathrm{~K} \alpha$

PI P $5 \mathrm{~K} \beta$

PIP $5 \mathrm{~K} \gamma$

PIP4 K

P I P $5 \mathrm{~K} \alpha$

PI P $5 \mathrm{~K} \beta$

PIP $5 \mathrm{K \gamma}$

PI P 4 K

P I P $5 K \alpha$

PIP $5 K \beta$

PIP $5 \mathrm{K \gamma}$

PIP $4 \mathrm{~K}$

PI P $5 \mathrm{~K} \alpha$

PIP $5 K \beta$

PIP5Ky

PI P 4 K

P I P $5 K \alpha$

PIP $5 K \beta$

PI P 5 K

PIP $4 \mathrm{~K}$

PI P $5 \mathrm{~K} \alpha$

PIP $5 K \beta$

PIP5K

P I P 4 K PI P $5 K \alpha$ PI P $5 \mathrm{~K} \beta$ PIP $5 K \gamma$
MASAS SGP S S SVGFS SFDPAVPSCTLS SAASGI KRPMA SEVLEARODSYI SLVPYASGMPIKKIGHRSVD - - - - - - - - - - - - - - - - - - - - - - - - - - - - - - - - - - - - - - MS SAAENGEAAPGK (1) AOPG GHGKKLGHRGVD $\& 1 \& 2 \& 3$ \&4 \#1

$----K L F R--A S E P I L S V L M W G V N H T I N E L S N V P V P V M L M P D D F K A Y S K I K V D N H L F N K--E N L P S R F K F$ S SGETTYKKTT S SALKGAIQLGITHTVGSLSTKPE-RDVLMQDFYVVES I FFP SEGSNLTPAHHYNDFRF ONEEKTYKKTAS SAI KGAIQLGI GYTVGNLT SKPE-RDVLMQDFYVVESVFLP SEGSNLTPAHHYPDFRF ASGETTYKKTTSSTLKGAIQLGI GYTVGHLS S KPE-RDVLMQDFYVVES I FFP SEGSNLTPAHHFQDFRF \#2 KEYCPMVFRNLRERFGIDDQDYQNSVTRSAP INSDSQGRCGTRFLTTYDRRFVIKTVS SEDVAEMHNI LK KTYAPVAFRYFRELFGIRPDDYLYS LCSEPLIELCS SGAS GS LFYVS S DDEFI IKTVQHKEAEFLQKLLP KTYAP LAFRYFRELFGI KPDDYLYS I CSEP LI ELSN P GAS GS LFFVT SDDEF I IKTVQHKEAEFLQRLLP KTYAPVAFRYFRELFGIRPDDYLYS LCNEP L I ELSN P GAS GS LFYVT S DDEF I IKTVMHKEAEFLQKLLP \&6 878 \#6

KYHQFIVECHGNTLLPQFLGMYRLTVDGVETYMVVTRNVESHRLTVHRKYDLKGSTVAREAS DKEKAKDL GYYMNLNQ-N P RT LLP KFYGLYCVQAGGKNIRIVVMNN LLPRSVKMHI KYDLKGSTYKRRASQKEREKPL GYYMN LNQ-N R RTLL KFYGLYCMQS GGINIRIVVMNNVLPRSMRMHFTYDLKGSTYKRRASRKEREKSN GYYMN LNQ-NPRTLLP KFYGLYCVQS GGKN IRVVVMNN I LPRVVKMHLKFDLKGSTYKRRAS KKEKEKS F \#7 \$2

PTFKDNDFLNE-GQKLHVGEESKKN FLEKLKRDVEFLAQLKIMDYSLLVGI HDVDRAEQEEMEVEERAED PTFKDLDFLQD I P DGLFLDADMYNALCKTLQRDCLVLQSFKIMDYSLLMS I HN IDHAQREPLSSETQYSV PTFKDLDFLQDMHEGLYFDTETYNALMKTLQRDCRVLES FKIMDYSLLLGIHFLDHS LKEKEEET PQNVP PTYKDLDFMODMPEGLLLDADTFSALVKTLORDCLVLES FRIMDYSLLLGVHN IDOHEREROAOGAOSTS

EECENDGVGGNLLCSYGTPPDSP GNLLSFPRFFGP GEFDPS--VDVYAMKS HESS PKKEVYFMAI IDI LT DT----RRPAPQKALYSTAMES-----IQGEARRGGTMETD--DHMGGI PARNSKGERLLLYIGI IDI LQ DA--- KRTGMQKVLYSTAMES-----IQGP GKS GD GI ITENPDTMGGI PAKS HRGEKLLLFMGI IDI LQ DE----KRPVGQKALYSTAMES-----IQGGAARGEAI ESD--DTMGG I PAVNGRGERLLLH I GI IDI LQ \#8

PYDTKKKAAHAAKTVKHGAGAEI STVNP EQYSKRFNEFMSNILT SYRFVKKLEHSWKALVHDG-DTVSVHRP GFYAERFQRFMCNTVF SYRLMKKLEHSWKALVYDG-DTVSVHRP SFYADRELKEMN SRVF SYRFIKKLEHTWKALVHDG-DTVSVHRPSFYAEREFKFMSNTVF

$450 / 562$

$396 / 540$

$444 / 668$

$\begin{array}{llll}\text { PIP4K } & \text { PIP5K } \boldsymbol{\alpha} & \text { PIP5K } \boldsymbol{~} & \text { PIP5K } \\ \text { \$1 Lys150 } & \text { \$1 Lys194 } & \text { \$1 Lys138 } & \text { \$1 Lys188 } \\ \text { \$2 Asp278 } & \text { \$2 Asp322 } & \text { \$2 Asp266 } & \text { \$2 Asp316 } \\ \text { \$3 Asp369 } & \text { \$3 Asp404 } & \text { \$3 Asp350 } & \text { \$3 Asp398 } \\ \text { \&1 Lys72 } & \text { \#1 Arg138 } & \text { \#1 Arg82 } & \text { \#1 Arg132 } \\ \text { \&2 Lys76 } & \text { \#2 Lys140 } & \text { \#2 Lys84 } & \text { \#2 Lys134 } \\ \text { \&3 Lys78 } & \text { \#3 Lys199 } & \text { \#3 Lys125 } & \text { \#3 Lys193 } \\ \text { \&4 Arg92 } & \text { \#4 Lys206 } & \text { \#4 Lys132 } & \text { \#4 Lys200 } \\ \text { \&5 Lys165 } & \text { \#5 Lys237 } & \text { \#6 Arg184 } & \text { \#5 Lys231 } \\ \text { \&6 Lys166 } & \text { \#6 Arg240 } & \text { \#7 Lys263 } & \text { \#6 Arg234 } \\ \text { \&7 Arg188 } & \text { \#7 Lys319 } & \text { \#8 Arg379 } & \text { \#7 Lys313 } \\ \text { \#1 Lys94 } & \text { \#8 Arg433 } & & \text { \#8 Arg427 } \\ \text { \#2 Lys96 } & & & \\ \text { \#7 Lys275 } & & & \end{array}$

Figure S2. Sequence alignment of the PIP4K and PIP5K proteins and relevant sequence patterns. The alignment of the amino acid sequences of the three PIP5K isoforms to the PIP4K sequence obtained using the Needleman-Wunsch global alignment algorithm (Needleman and Wunsch, 1970). The catalytic residues shared by PIP4K and PKA (Rao et al., 1998) and found to be conserved in the alignments of the PIP5Ks are highlighted in red (and marked with \$). Positively charged residues contributing to the positive surface potential of PIP4K and not conserved in PIP5K (Fig. 3) are highlighted in blue (and marked with \&). Positively charged residues contributing to the positive surface potential of the PIP5K proteins are highlighted in green (and marked with \#). Three of the latter residues are also conserved in PIP4K (K94, K96, and K275). 
a
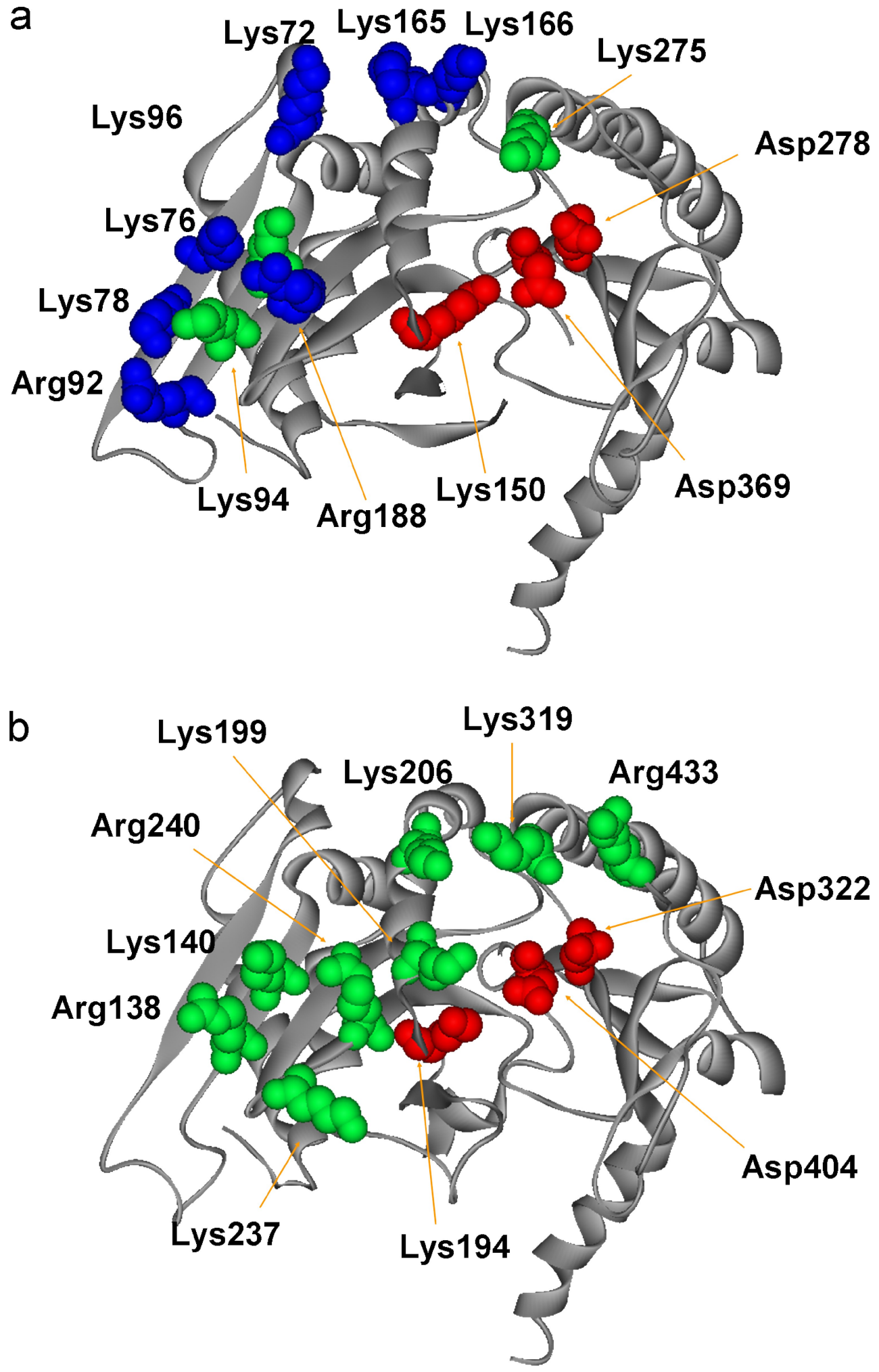

Figure S3. Position of catalytic and positively charged residues of the PIP4K and PIP5K proteins. (a) Catalytic and positively charged residues mapped onto the three-dimensional structure of PIP4K. The protein backbone is depicted as a gray ribbon. Highlighted side chains are shown in full atomic detail with the coloring scheme following that of Fig. S2. (b) Catalytic and positively charged residues mapped onto the three-dimensional structure of PIP5K- $\alpha$. The backbone and side chain representation and the residue-coloring scheme is the same as in a. Interestingly, PIP4K and PIP5K both harbor a very similar number of positively charged side chains (PIP4K, 10; PIP5K, 10-11) on the flat face. 

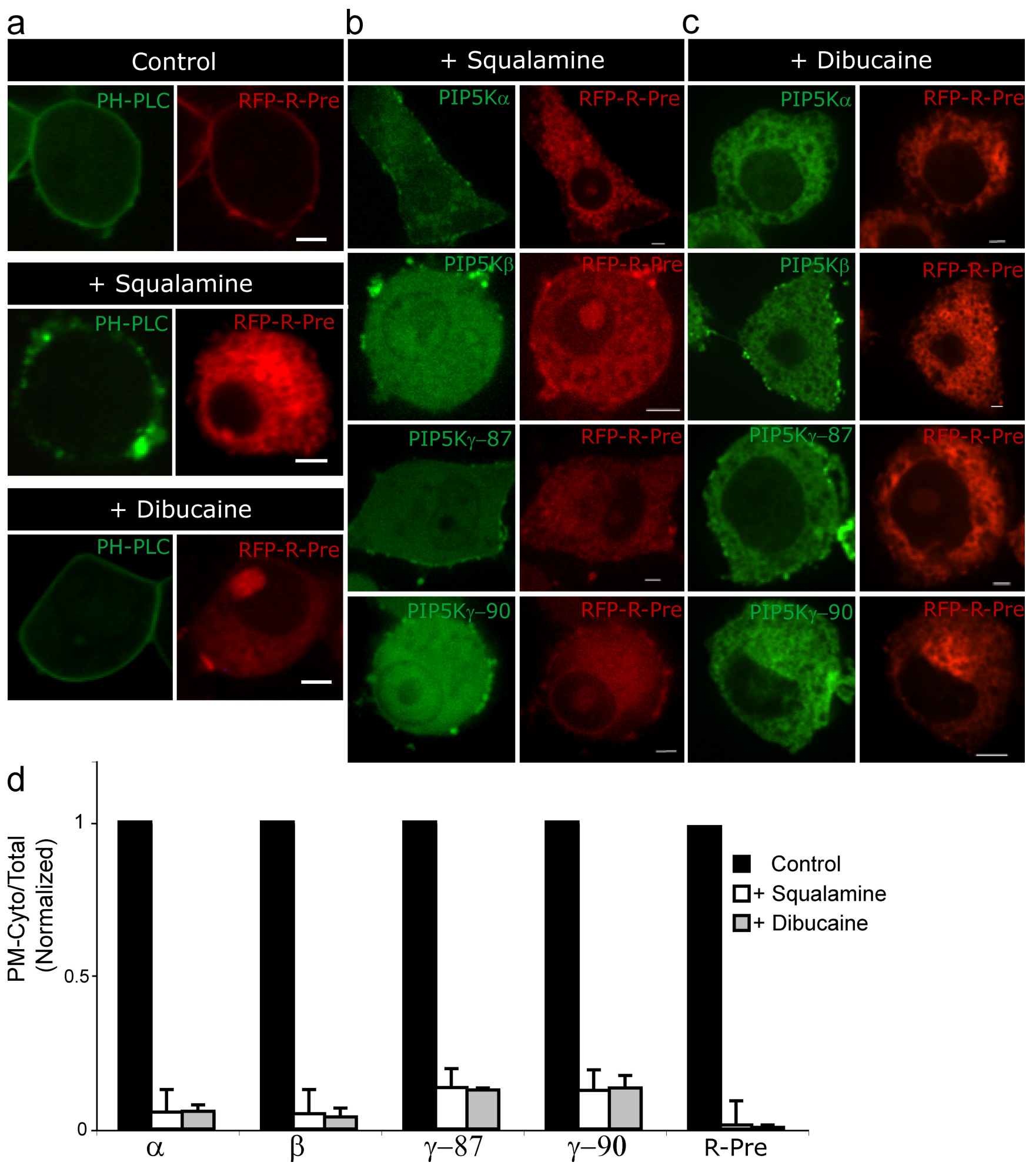

Figure S4. Localization of PIP5Ks is altered by a reduction in membrane surface charge. (a-c) Macrophages were cotransfected with either PH-PLC- $\delta$ (a) or the specified GFP-PIP5Ks (b) and the surface charge sensor R-Pre. Images were acquired after treatment with $1 \mathrm{mM}$ dibucaine for $20 \mathrm{~min}$ or with $5 \mu \mathrm{gg} / \mathrm{ml}$ of the dicationic sterol, squalamine, for $5 \mathrm{~min}$. (d) Quantification of the effects of the treatment on the distribution of the specified PIP5K and R-Pre. Data are means $\pm \operatorname{SEM}(n>30)$. Bars, $3 \mu \mathrm{m}$. 


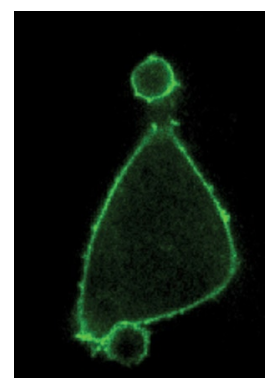

Video 1. Distribution of PIP5K- $\gamma \mathbf{8 7}$ during phagocytosis. RAW macrophages were cotransfected with PIP5K- $\gamma 87-G F P$ and RPre. Phagocytosis was initiated by exposure to IgG-opsonized latex beads. The green fluorescence of PIP5K- $y 87-G F P$ is shown in this video, whereas the corresponding red fluorescence is shown in Video 2.

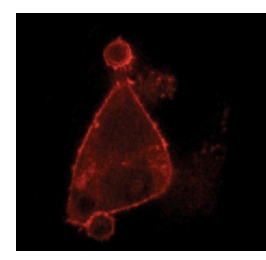

Video 2. Distribution of R-Pre during phagocytosis. RAW macrophages were cotransfected with PIP5K-y87-GFP and R-Pre. Phagocytosis was initiated by exposure to lgG-opsonized latex beads. The red fluorescence of R-Pre is shown in this video, whereas the corresponding green fluorescence of PIP5K-y87-GFP is shown in Video 1.

\section{References}

Laskowski, R.A., M.W. MacArthur, D.S. Moss, and J.M. Thornton. 1993. PROCHECK: a program to check the stereochemical quality of protein structures. J. Appl. Cryst. 26:283-291.

Needleman, S.B., and C.D. Wunsch. 1970. A general method applicable to the search for similarities in the amino acid sequence of two proteins. J. Mol. Biol. 48:443-453.

Rao, V.D., S. Misra, I.V. Boronenkov, R.A. Anderson, and J.H. Hurley. 1998. Structure of type IIbeta phosphatidylinositol phosphate kinase: a protein kinase fold flattened for interfacial phosphorylation. Cell. 94:829-839. 